\title{
The Spectrum and Distribution of Imaging Findings at Coronary Computed Tomography Angiogram in Patients Suspected to Have Coronary Artery Disease: A Cross-sectional Study Carried Out at Plaza Imaging Solutions in Nairobi, Kenya
}

\author{
Omamo Eunice A. \\ Department of Diagnostic Imaging and Radiation Medicine, University of Nairobi, Kenya
}

Copyright@2017 by authors, all rights reserved. Authors agree that this article remains permanently open access under the terms of the Creative Commons Attribution License 4.0 International License

\begin{abstract}
Background: Coronary CT angiography (CCTA) has in the recent past evolved rapidly due to development of sophisticated multi detector row CT (MDCT). The cardinal indication for CCTA is the evaluation of suspected or known coronary artery disease (CAD). Coronary CTA imaging can detect and characterize atherosclerotic plaques. In addition, it can confirm or exclude luminal stenosis. Other applications include identification and assessment of variations in coronary arterial circulation patterns, evaluation of isolated anomalous coronary vessels, by-pass grafts, coronary stents patency and assessment of left ventricular function. Study objective: This study was set to determine the spectrum, pattern and distribution of imaging findings at Coronary CT angiograms as detected by a 320 row MDCT in patients who had undergone coronary CTA. Study design: A cross sectional study. Setting: Plaza Imaging Solutions Limited, Nairobi, Kenya. Subjects: Patients imaged at Plaza Imaging Solutions Limited over a period of 6 months. Materials and methods: Study subjects: Consecutive patients referred for coronary CTA at Plaza Imaging Solutions Limited and met the inclusion criteria. Ethical considerations: Ethical approval and clearance was obtained from the KNH-UON Ethics Review Board. Written and verbal consent was obtained from the participating persons and/or their kin Tools: A two-part data collection form was used to manually record all the relevant data. The information included socio-demographics, clinical and imaging finding of Coronary CTA. A low dose calcium scan and a standard coronary CTA were performed. Each Coronary CTA study was reviewed by a radiologist together with the researcher. Statistical analysis: Data analysis was performed using the Statistical Package for Social Sciences (SPSS) employing relevant statistical tests. The results were presented in form
\end{abstract}

of frequency tables, graphs and charts and discussed accordingly. Statistical significance was set at $\mathrm{p}<0.05$. Results: During the six-month period, 38 out of a total of 42 scanned patients were recruited into the study. The age distribution ranged from 21 to 89 years with a mean age of 56 years (SD 14.8-19.2). Male to female ratio was 5:4. Chest pain (angina) accounted for the commonest indication for imaging at $63.2 \%$. Risk factors of hypertension and diabetes mellitus were noted in $39.5 \%$ of patients. More males reported a co- morbid state of diabetes mellitus (DM-Type II) while more females $50 \%$ had hypertension. Overall there were more patients with abnormal scans at $76 \%$ than the normal. Atherosclerotic coronary plaque lesions were the most commonly encountered abnormality accounting for 93.1\%. The predominant plaque type was the non-calcified plaque seen in $50 \%$ of males and $61.5 \%$ of females. The total calcium scores (Agatston) ranged from 0 to 242. Calcified plaques were predominantly on the left anterior descending coronary artery (LAD), while the non-calcified plaques were significant on the right coronary artery (RCA). Conclusion: The study demonstrated common occurrence of coronary atherosclerotic plaques in the older age group with lower incidence seen in younger individuals aged less than 40 years of age. It also demonstrated the wide spectrum of luminal effect caused by the atherosclerotic plaques. Furthermore, it demonstrated the paucity of calcified atherosclerotic plaques amongst the local population undergoing coronary CTA highlighting the insignificant nature of coronary calcium score in this setting. Recommendations: Based on the findings and conclusions of this study, a recommendation is made for coronary CTA to be performed in the older age group of $>55$ years of age with symptomatic features of ischemic heart disease. Coronary calcium scoring is not necessary in individuals undergoing coronary CTA in this 
setting (black race) as no correlation is found between total calcium score and atherosclerotic disease burden.

Keywords Coronary CT Angiography (CCTA), Coronary Artery Disease (CAD), Coronary CT Angiogram (CTA)

\section{Background}

Ischemic heart disease (IHD) is a generic term used to denote a spectrum of clinical disorders of the heart resulting from an imbalance between myocardial need for oxygen on the one hand and the adequacy of oxygen supply on the other. Because reduction in coronary blood flow almost always is the cause of the imbalance, IHD is also known as coronary artery disease. This description obviously and directly illustrates the actual cause of coronary ischemia arising from compromise of the coronary arteries $(1,2)$.

Coronary artery disease is the result of narrowing of the coronary blood vessels lumen. These vessels, the coronary arteries and their branches supply the heart structures including the myocardium with oxygen and nutrients. The luminal narrowing is caused by a buildup of atherosclerotic plaques on the inner walls of the coronary vessels resulting in gradual or abrupt reduction or complete cessation of blood supply to the heart structures. The resultant reduction in blood supply to the myocardium and its components due to atherosclerotic plaques causing luminal narrowing may either be symptomatic or asymptomatic, occur during activity or at rest and may ultimately result in a myocardial infarction or sudden death. $(1,2,3)$.

Worldwide, atherosclerosis has an enormous impact on the global economics and health exerting a burden as a leading cause of morbidity and death amongst both men and women. (4). Coronary artery disease is the leading cause of death in the developed world with a prevalence of approximately $7.0 \%$ (5). Despite significant advances in the diagnosis and management of CAD in the developed world, it remains the leading cause of death in the United States and generally worldwide.

The prevalence may be rising in sub-Saharan Africa due to rapidly changing trends in urbanization, lifestyle, sophisticated technological acquisitions and probably better imaging tools but data is still lacking (6).

Risk factors play a key role in the timing, initiation and rate of progression of coronary atherosclerosis plaque development and therefore in prevention of the disease. The Framingham study has been able to provide insight regarding risk factors numbering more than 300 but most of which are interdependent and the number of truly independent risk factors remains small (7).

As stated earlier, sudden death is the initial presentation of CAD in $50 \%$ of men and $64 \%$ of women past the middle age. The viable, available and non-invasive strategy for reducing mortality in these patients is through primary prevention (8, 9).

How can primary prevention be thus achieved? The answer may lie in evaluating and identifying the supposedly 'minimal risk' group for further evaluation. The presumably 'low cardiovascular risk' group is the target population for atherosclerosis imaging which has been proposed as a strategy for the early and more accurate identification of individuals at risk for CHD 'hard events'. These individuals once identified are likely to derive the greatest benefit from lifesaving strategies. $(8,9)$.

Cholesterol and calcium phosphate accumulate in the coronary arterial walls in different proportions resulting in soft, fibrotic or calcified atherosclerotic plaques. These plaques together with resultant vessel stenosis can easily be detected using radiological means such as MDCT, EBCT and conventional angiography.

Newer, faster MDCT scanners with higher spatial and better temporal resolutions are capable of cardiac imaging in a single heartbeat. MDCT is the current imaging modality of choice used in coronary atherosclerotic plaque detection and characterization.

The scanners are capable of accurately and precisely demonstrating calcified and non-calcified plaques.

In addition, coronary $\mathrm{CT}$ angiography can demonstrate the coronary arterial tree pattern showing coronary vessel dominance, arterial variants and isolated anomalous arteries in addition to other coronary vessel anomalies such as myocardial bridging, coronary aneurysms and fistulas.

During the current study, the MDCT scanner used, Aquilion 1 can acquire up to 320 tomograms per rotation depending on the number of detector rows activated during the scanning session. The number of images generated also depends on other settings used during the actual image acquisition such as the selected slice thickness which differs for calcium score, functional cardiac imaging and coronary vessel angiogram.

Following image acquisition, the volume data sets are automatically processed by the CT scanner using modern processing and post processing methods including reconstruction of the images in distinct phases.

Image presentation for interpretation by the radiologist is received on a Vitreaworkstation ${ }^{\mathrm{TM}}$. This is an intuitive multi-modality image manipulation and visualization software which enables images to be manipulated further to amongst other things view the acquired images in 2D, 3D and 4D, perform multi-planar and curved planar reformations for accurate assessment of coronary vessel courses and stenoses, analyze coronary vessel wall, lumen and plaque composition using the in-built software amongst other image manipulative possibilities.

\section{Literature Review}

\section{Coronary Artery Disease}


Coronary artery disease is also known as ischemic heart disease or atherosclerotic heart disease. It is the epidemic of our time and is set to remain so for the foreseeable future. Coronary artery disease is the most common of all cardiovascular illnesses being the second most common non-communicable disease in Kenya (10).

Atherosclerosis is a word derived from the Greek term 'athera-' meaning porridge and '-scleros' meaning hard. Coronary artery atherosclerosis is an intimal disease (though associated changes are seen within the tunica media and adventitia) affecting the epicardial muscular arteries. It is characterized by deposition of soft cholesterol containing material and hardening of the intimal layer of the arterial wall (11).

Coronary artery disease remains responsible for one third deaths in individuals over the age of 35 years in the developed world and its manifestations have an enormous impact on the quality of life. No epidemiological data to this effect is available in Kenya. Although the rates have declined over the last couple of years in the developed world, the trends are reversed in the developing countries with the prevalence of lifestyle diseases rising. Though data is still lacking to illustrate the situation in sub Saharan Africa and indeed the whole of the developing world, the situation may not be different $(5,6)$.

Coronary artery disease is a slow chronic process with a lengthy incubation period, during which biologic risk factors interact with genetic and environmental influences to initiate and promote the development of atherosclerotic plaques (1, 8). It is postulated to begin in the second decade of life and slowly progress throughout life with the rate of progression pegged on independent risk factors such as family history of $\mathrm{CAD}$, age, gender and race.

Modifiable risk factors such as cigarette smoking, Diabetes mellitus, and hypertension among others determine disease severity and acceleration once it occurs.

Atherosclerosis is the only vascular disease known to be associated with coronary arteries calcification (12). Atherosclerotic plaque proceeds structurally through multiple phases during which the characteristic features of the plaques change. Initially the plaques result from deposition of cholesterol on vascular walls. During this early phase, the soft plaque is described as unstable and may rupture or ulcerate causing acute thrombus formation. This thrombotic process occurs because of luminal blood interacting with the clot inducing soft plaque material often resulting in complete occlusion of vessel lumen.

Plaques subsequently become fibrotic over a course of time before eventually becoming calcified. This subsequent calcification of a formerly soft plaque is regarded as a protective mechanism instituted by the body to provide stability to an otherwise unstable lesion prone to cause thrombosis and therefore vessel occlusion. A calcified plaque is therefore considered stable, while a non-calcified i.e. a soft plaque is unstable and prone to rupture and thrombus formation with a higher incidence of coronary hard events (13).

The relationship of CAC to obstructive atherosclerotic disease has been extensively investigated and documented. While the presence of CAC is nearly $100 \%$ specific for atherosclerosis, it does not necessarily indicate obstructive disease since both obstructive and non-obstructive lesions may have calcified atherosclerotic plaques present in the walls (14). It is therefore the co-occurrence of both the calcified and non-calcified plaques that provide the means of estimating the probability of occurrence of acute coronary events. (13)

Furthermore, studies have indicated that although CAC detection and measurement cannot localize a stenotic lesion or one prone to rupture, calcium scoring may be able to predict a patient's CAD event risk by the strong association between calcified plaques and coronary atherosclerotic disease burden $(9,13)$. However, not all plaques are purely calcified with the majority being either 'soft' or mixed plaques. In a histological study, the total calcium area was found to be approximately $20 \%$ of the total atherosclerotic plaque area $(13,15$,) indicating the minimal extent of calcification within a plaque. The extent of coronary calcification is however directly related to the volume of the atherosclerotic plaque in question. (13)

\section{Coronary Atherosclerosis Imaging}

Coronary heart disease has traditionally been imaged using conventional catheter angiography which over time has come to be been regarded as the 'Gold' standard in coronary imaging.

However, with the rapid innovation and application of modern technology, non- invasive means such as the MDCT coronary CT angiography can be used as reliably as conventional coronary angiography in the detection of significant obstructive coronary artery disease and is replacing conventional angiography unless interventional procedures are planned.

MDCT scanners with a minimum of 64 detector rows can satisfactorily perform coronary CT angiography with comparable spatial and temporal resolution. The volume data sets acquired during the scanning tend to get better with higher orders of detector rows giving better reconstructed images and better spatial resolution of the distal coronary arterial branches $(16,17)$.

Dual energy source CT scanners have an added advantage over the sole source scanners in terms of achieving higher temporal resolution. This possibility is particularly advantageous in patients with higher heart rates or arrhythmias since the heart rate does not have to be slowed down considerably as is needed for the sole source scanners ( $<65-70$ beats per minute for sole source compared to 85-90 for dual source (18).

Common indications for coronary CT angiography include atypical angina, typical angina with inconclusive stress test, and presence of risk factors and high risk of major 
coronary events, proximal stent patency follow up and by-pass patency follow up (19).

In selected patients with appropriate indications, coronary CTA may eventually replace conventional angiography as the first line imaging modality of choice.

With CT, coronary angiography, calcified, mixed and non-calcified atherosclerotic plaques can be accurately visualized as well as the evaluation of vessel luminal stenosis. Initial studies have shown that certain plaque characteristics such as very low CT attenuation are associated with plaque instability and therefore vulnerability to cause cardiac events (20).

Previously used 16 and 64 slice MDCT have shown encouraging sensitivities and specificities in relation to the detection of significant coronary artery stenoses defined as more than $50 \%$ reduction of arterial lumen. Data shows that 64 detector row scanners when used for coronary CT angiography in patients with suspected CAD, had study sensitivity and specificity of $94 \%$ and $92 \%$ respectively. In the same breath, the negative predictive value for a negative scan stood at $99 \%$ which translated to an applicable role of coronary CTA to rule out the presence of stenoses in selected patient populations (21). Similar or more refined findings can be extrapolated for higher slice scans.

\section{Technical and Physical Perspectives of Coronary CT Imaging}

\section{Historical Perspective}

The first CT scanner was introduced by Hounsfield in 1973 being the starting point of integration of CT imaging into the medical practice. The very early scanners had relatively long gantry rotation time of up to 5 seconds. This only enabled rough visualization of the cardiac morphology due to the very poor temporal resolution.

In the early 1980s EBCT was introduced. This had a very high temporal resolution of up to $100 \mathrm{~ms}$ permitting motion-free cardiac imaging even at high heart rates. It allowed visualization of coronary arterial calcification and therefore enabled calcium scoring.

The 4-slice MDCT scanners made headway in the late 1990s and improved scanning speeds making feasible scanning of the heart in one breath hold. Image quality improved further due to the impact of 16 slice MDCT introduced in 2001 which considerably eliminated motion artifact inherent to the heart.

The introduction of 64-slice MDCT permitted simultaneous acquisition of 64 slices enabling scanning of the entire coronary tree in less than 13 seconds with iso-volumetric spatial resolution.

Dual source CT scanners were an even better innovation approach that allows further decrease in temporal resolution to achieve good image quality at higher heart rates of up to 85-90 beats per minute.
Now with the recent introduction of 320- slice MDCT scanners, the imaging of coronary vessels has evolved enabling clear visualization of atherosclerotic plaques and stenoses up to the third order branches significantly improving the diagnostic quality of the images acquired.

\section{The MDCT Technology}

The MDCT scanner is a technological development that employs a rotating gantry and variable numbers of detectors ranging from 4 to 320. MDCT scanners use rapidly rotating $\mathrm{X}$-ray tubes and several rows of detectors also rotating. The tube and detectors are fitted with slip rings allowing them to perform continuous movements through 360 degree rotations without tangling up of cables.

While the gantry continuously rotates, the table may or may not move through the imaging plane at a predetermined speed. This relative speed of the gantry rotation compared to table motion is what constitutes the scan pitch. With the higher number of detectors, the width of the heart can be comfortably scanned using a single gantry rotation with a single breath hold.

Speed and time are of essence in imaging of the heart due to the inherent non-linear cardiac motion. The newer MDCT present markedly improved spatial and temporal resolution with these being as low as $0.4 \mathrm{~mm}$ and $0.75 \mathrm{msec}$ respectively. Present versions complete a 360-degree rotation in about $4 / 10^{\text {th }}$ of a second.

These exposure times can be reduced by utilizing only a portion of the 360 degree to as little as 0.2 of a second.

Newer higher slice $>64$ MDCT with a temporal resolution of $<$ msec seconds and as little gantry rotation time as 0.33 seconds can image the heart in very limited time with no need to slow the heart rate using pharmacological means.

The cephalad-caudad length of the heart can be captured in one heart beat at the high spatial resolution of modern MDCT scanners.

MDCT provides thin tomographic images of the heart. It has both high spatial resolution of computed tomography and good temporal resolution as was the case with the EBCT. Though the radiation exposure is not comparable, lowering radiation dose has been the goal of modern CT manufacturing companies which has seen the progressive drop in radiation doses from more than $25 \mathrm{mSv}$ for 4 slice CT through $10-\mathrm{mSv}$ for 64 slice and now to less than $5.5 \mathrm{mSv}$ for the high-end superfast CT scanners.

\section{Coronary Arteries}

The major coronary arteries together with their second order branches can usually be well visualized on images acquired on MDCT during coronary CT angiography. Third order branches may be visualized, but smaller branches are generally not visible because of their small size and limited spatial resolution $(22,23)$.

In the commonly occurring normal anatomy, the main 
coronary arteries arise from the proximal aorta at the right and left sinuses of Valsalva respectively. The posteriorly situated non-coronary sinus gives no rise to any vessel (22, 23).

The main left and right coronary arteries segments course in the left and right atrio-ventricular grooves between the atria and the ventricles and then perpendicularly in the anterior and posterior inter-ventricular grooves between the left and the right ventricles respectively. The coronary arteries and their side branches vary considerably in terms of their presence or absence, size, shape and length $(22,23)$.

The right coronary artery (RCA) arises from the aorta at the right sinus of Valsalva and courses in the right atrio-ventricular groove. Acute marginal branches arise from the mid-segment and the posterior right ventricular branches from the distal segment.

The left main (LM) coronary artery arises from the aorta at the left sinus of valsalva and a short distance later bifurcates into the left anterior descending (LAD) and the left circumflex artery. In one third of the population, the left coronary trifurcates with an intermediate branch (IMB) called the ramus medianus. In about $1 \%$ of the population the left main coronary artery is absent and there are separate ostia for the LAD and the LCX

Normal coronary arteries have strong walls composed of three layers namely the tunica interna, tunica media and tunica adventitia.

The tunica interna is an endothelium layer with a basement membrane that is virtually in contact with the flowing blood within the arterial lumen.

The tunica media is a thick middle layer of smooth muscle and elastic fibers that responds to the cardiac cycle of systole and diastole and thus maintains the active flow of blood through the vessel.

The tunica externa is the outer connective tissue layer composed principally of elastic and collagen fibres.

\section{Coronary Artery Variants and Anomalies}

Studies previously carried out indicate that anatomical variants of the coronary artery tree are very frequent and numerous (23).

Coronary arterial dominance is defined as the main coronary vessel that gives rise to the posterior descending artery which supplies the posterior portion of the left ventricular septum.

According to Cademartiri F. et al., right dominance was found to be the most common occurrence with a prevalence of approximately $86.6 \%$. Left dominance was noted to be approximately $9.2 \%$ while co- dominance is approximately $4.2 \%$ (23).

Several studies observed a prevalence of about $18.4 \%$ of coronary variants and anomalies. Patients presented with either a single or multiple coronary anomalies.

Isolated coronary anomalies are uncommon in the general population as opposed to the coronary anomalies that occur as part of a hereditary spectrum or as a manifestation of congenital heart disease (24).
Most isolated coronary artery anomalies are detected incidentally as they remain relatively asymptomatic throughout life though early detection and evaluation are essential. This is because of their potential association with myocardial ischemia and sudden death as detected at autopsy especially in athletes with sudden death.

The anomalous origin and course of a coronary artery is less frequently encountered with a very low prevalence of less than $1 \%$ of the imaged population.

The known isolated coronary artery anomales include anomalies of artery origin such as:

1. Single coronary artery

2. Anomalous origin of the left coronary artery from pulmonary artery (ALCAPA) resulting in the left ventricle being perfused by relatively desaturated blood under low pressure leading to myocardial ischemia.

3. Inter-arterial left coronary artery: Termed malignant inter-arterial LCA with the LCA arising from the right sinus and coursing between the aorta and the pulmonary artery

4. Origin from the non-coronary cusp $(24,25)$.

Less frequent anomalies include early take off of the posterior descending artery, retro-aortic LCX amongst others. Other isolated anomalies include coronary arterial duplication, coronary arterial aneurysms and myocardial bridging.

Myocardial bridging is commonly observed on the LAD artery though it may also be seen in the diagonal or IM branches. It is defined as the descent of a portion of the coronary artery into the myocardium. Coronary CTA can detect more cases of myocardial bridging with an observed rate of $30 \%$ compared to the less than $5 \%$ detected at interventional coronary angiography. This is due to the improved depiction of myocardial tissue that is achieved by MDCT coronary angiography.

The depth of the bridging myocardium is more important and of more clinical significance compared to the length of the bridged vessel. Debate is still on going on the hemodynamic significance of this anomaly (23-25).

Coronary aneurysms defined as more than $1.5 \mathrm{~mm}$ if compared with the normal vessel diameter have been found to have a prevalence of $1.6 \%$ of studied populations (23).

Anomalous terminations encompass coronary artery fistulas and extra-cardiac termination of coronary arteries. Coronary arterial branches may terminate into the right ventricle giving rise to left to right shunting of blood from the coronary vessels to the right side of the heart (23).

Previously and for a long time, premorbid diagnosis of these coronary anomalies was made at conventional angiography which is an invasive procedure with attendant complications and limited projection. MDCT is particularly superior to conventional angiography in its ability to delineate the ostial origin and proximal pathway of an anomalous artery (26).

\section{Non-Coronary and Extra Cardiac Findings}


In addition to the coronary findings, non-coronary but cardiac lesions likely to be encountered include undiagnosed congenital heart disease, cardiac valvular disease, cardiac masses, thrombi, tumors and pericardial disease.

Studies previously conducted indicate that extra-cardiac findings occur in approximately $10 \%$ to $60 \%$ of cases undergoing cardiac CT imaging depending on the patient population being studied. Although majority of these findings are benign without a need for follow up, some may be indeterminate while others may be clinically significant (16).

These studies conclude that thorough and methodical evaluation of Coronary CT angiography scans should be reinforced (16, 24, 25 and 27).

\section{Problem Statement}

The prevalence of coronary artery disease is about $7.0 \%$ worldwide. It is the leading cause of death in the developed world and the third most common cause of death in sub Saharan Africa (5, 6).

Nearly half of deaths related to CAD occur as a first-time presentation in patients unknown to be at risk for CAD events $(28,29)$.

This project documents the trend of atherosclerotic cardiovascular disease in the imaged individuals locally while outlining the spectrum of radiological findings at coronary CT angiography, therefore define the role of non-invasive angiography in the management of patients suspected to have CAD.

\section{Study Justification}

Coronary CT angiography is a relatively new diagnostic imaging technique in the field of cardiology and cardiovascular diseases management. Though well-grounded in the developed world as an essential work up for individuals with known or suspected atherosclerotic cardiovascular disease, this imaging techniques is yet to gain root in the developing world as a fast, easy, non-invasive and very informative one stop shop tool for rapid assessment of patients presenting with acute chest pain or coronary signs and symptoms.

The current study sought to outline the prevalence and severity of atherosclerotic coronary disease in individuals undergoing coronary CTA as identified by MDCT imaging.

No such study has been carried out in this part of the world and it would be important to assess the role of coronary CT angiography in the management of individuals suspected for CAD.

\section{Research question}

What is the spectrum of findings at coronary CT angiography in the imaged local population?

What is the prevalence and distribution of coronary atherosclerotic disease in the patient population undergoing Coronary CT angiography?

\section{Study Objectives}

\section{Broad Objective}

To determine the spectrum and distribution of coronary imaging findings at coronary $\mathrm{CT}$ angiography in patients suspected to have coronary artery disease.

\section{Specific Objectives}

1. To describe the clinical and socio-demographic characteristics of patients presenting for coronary CTA.

2. To determine the occurrence, type and distribution of atherosclerotic plaques on the coronary arterial tree.

3. To determine the distribution in the population of various coronary arterial tree patterns.

\section{Study Methodology}

\section{Study Design}

The current study was a descriptive cross sectional study conducted over a period of 6 months at Plaza Imaging Solutions Limited in Nairobi following approval by the KNH/UON- Ethics Research Committee.

\section{Study Area Description}

The study was carried out at Plaza Imaging Solutions Limited, a privately owned and run imaging facility within the city of Nairobi.

\section{Study Population}

The study population comprised of consecutive patients referred to the imaging facility for coronary CT angiography.

\section{Criteria}

Inclusion Criteria

Subjects were eligible to participate in the study if all the following conditions exist:

- Adult patients ( $>18$ years of age).

- Request form with a query for ischemic heart disease.

- Diagnostic quality coronary CT angiograms performed.

- Patient has given consent to participate.

\section{Exclusion Criteria}

Subjects were excluded from the study if any of the following conditions existed at the time of recruitment: 
- $\quad$ Patients sent for cardiac CTA for indications other than suspected ischemic heart disease. These included patients sent for exclusion of intracardiac thrombi and congenital heart disease evaluation.

- Poor quality non-diagnostic coronary CTA scans such as those with motional artifacts or reduced noise to signal ratios rendering readability unreliable

- Patients under 18 years of age undergoing cardiac CTA

- Patients who did not give consent for inclusion into the study

\section{Method of Performing Coronary CTA}

The patient is optimally prepared for the examination by avoiding caffeine containing drinks/foods at least 12 hours prior to scanning. The patient also fasts for at least 4 hours in lieu of the contrast media to be administered.

Once consent is obtained and adequate explanation pertaining to the examination procedure is offered the patient is placed supine on the scanner table with the arms above the head. He is not to move to ensure accuracy of scanning of the planned region of interest. ECG electrodes are placed at the respective positions on the anterior chest walls. If the resting heart rate is above 75 beats per minute, a beta blocker (Metoprolol $50 \mathrm{mg}$ oral) in this case is administered and the heart rate reviewed in 45 minutes to 60 minutes. Once the optimal resting heart rate of $60-65$ is obtained the examination is set in motion. The oral metoprolol was repeated one more time an hour after the initial dose in cases which the desired heart rate is not achieved in a single dose.

A large caliber IV (French Gauge 18) access is obtained preferably on the right cubital vein due to the shorter distance and direct course of the vein to the right side of the heart. Contrast administration on the right cubital vein also ensures least contrast dilution. The amount of contrast injected in bolus is $40-60 \mathrm{mls}$. Scanning is done by bolus tracking with the region of interest placed in the proximal descending aorta.

Aerosol nitroglycerine (2-3 puffs) equivalent to $0.8 \mathrm{mg}$ to $1.2 \mathrm{mg}$ is administered orally within 20 minutes of the angiography examination.

The scanning field extends from above the left atrium to immediately below the heart unless the clinical information of previous surgery /treatment requires tailoring of the scan length to cover bypass graft origins whether venous or arterial.

Breath-hold training is performed to ensure acceptable heart rate variability during scanning which should be less than $10 \%$ since greater variability will degrade image quality.

\section{Scanning Parameters}

Plaza Imaging Solutions Limited will use the CT scanner's preset Coronary CTA imaging protocol which is selected from the machine control panel by the radiology technician performing the scanning once the required study is correctly identified from the radiology requisition form.

Tube current abbreviated as $\mathrm{mA}$ is adjusted per individual patient's body weight ranging from 300 to 450 while the $\mathrm{kV}$ is maintained at $120 \mathrm{kv}$. The angiography examination is performed using retrospective ECG gating.

The reconstruction parameters such as the algorithm to be used and reconstruction thickness are pre-selected as $0.5 \mathrm{~mm}$ with a reconstruction increment of $0.4 \mathrm{~mm}$ for coronary vessels. Reconstruction for left ventricular function is chosen at $1 \mathrm{~mm}$ thickness and $3 \mathrm{~mm}$ for calcium scoring. Reconstructions are done after ECG editing following identification of the minimal cardiac motion phases. Reconstructions are done through the cardiac cycle at $10 \%$ intervals resulting in 10 phases.

The amount and flow rate of the iodinated contrast media is adjusted to the patient's body weight and the bolus tracking method with automatic trigger for scanning used to determine the total quantity of iodinated water soluble contrast media for the coronary angiogram examination.

\section{Calcium Score}

A default non-enhanced calcium score scan is run with a slice thickness of $3 \mathrm{~mm}$ and calcium scoring is performed on the Vitrea workstation. The total calcium score termed the Agatston score is automatically generated by the software after identification and detection of calcified atherosclerotic plaques. Calcium scoring was done semi automatically through identification of calcified plaques which were then summed up to give a total calcium score termed the Agatston score.

\section{Agatston Score Defined}

Agatston score is a measure of calcium used to denote the calcified plaque burden from a non-enhanced coronary CT examination. It is named after Dr. Arthur Agatston and was initially based on findings at EBCT. The score is calculated by the processing software (in the current study done by Vitrea processor) using a weighted value assigned to the highest density of calcification measured in Hounsfield units. The weighted values are assigned as thus; 1 for 130-199 HU, 2 for 200-299 HU, 3 for 300-399 and 4 for 400 and any greater value. The weighted score is then multiplied by the area in $\mathrm{mm}^{2}$ of the calcified portion of the coronary plaque to give a score. This scoring is done for each of the main coronary vessels and the identifiable branches. The sum of the scores for all the vessels are compiled and given as the total Agatston score.

\section{Plaque Characterization}

Plaque characterization and degree of luminal stenosis were determined using in-built software in the Vitrea workstation. 
Plaques characterization included classification of plaques into soft, fibrotic and calcific depending on the Hounsfield units of the identifiable and detectable plaque. The workstation additionally has color coding for the different types of plaques with red, purple and yellow colors representing soft, fibrotic and calcified plaques respectively.

The Hounsfield unit representing soft plaque is -100 to +60 , fibrotic plaques +60 to 130 and calcified plaques $>140$ $\mathrm{HU}$.

The Vitrea workstation can calculate the severity of luminal stenosis as a percentage of both the unaffected diameter and area. It likewise gives the tortuosity index

\section{Sample Size Determination}

The numbers of coronary CT angiogram examinations conducted at Plaza Imaging Solutions are limited. Thus, the formula for estimating a population proportion shown below will be used with a finite population correction to determine the sample size:

$$
\mathrm{n}=\frac{N Z^{2} P(1-P)}{d^{2}(N-1)+Z^{2} P(1-P)}
$$

Where:

$\mathrm{n}=$ sample size with finite population correction

$\mathrm{N}=$ population size (approximately 7 imaging examinations conducted at the imaging center monthly yielding a population size of 42 for the 6-month study period)

$\mathrm{Z}=\mathrm{Z}$ statistic for a level of confidence (95\% confidence $=1.96$ )

$\mathrm{P}=$ expected proportion of 0.5 , and

$\mathrm{d}=$ precision (set at 0.05 )

$$
n=\frac{42 \times 1.96^{2} \times 0.5(1-0.5)}{0.05^{2}(42-1)+1.96^{2} \times 0.5(1-0.5)}
$$

$\mathrm{N}=38$

The coronary CT angiography scans was performed using the laid down protocol for the cardiac CTA and the examination reported by a radiologist.

\section{Sampling Method}

During a 6-month period a cohort comprising consecutive patients/clients referred for coronary CTA at Plaza Imaging Solutions Limited was recruited after obtaining informed consent.

\section{Recruitment and Consenting}

Recruitment took place at the imaging facility's CT scan waiting room and was done by the principal investigator or research assistant. The criteria for recruitment entailed checking the age and clinical indication for the coronary CTA. Consecutive patients were screened for inclusion and subsequently recruited.

Informed consent was both verbal and written, taken by the principal investigator or research assistant from the patient or the next of kin for those unable to give consent following an explanation on the procedure of examination involved including the risks and benefits.

\section{Data Collection}

Data collection was done manually by use of a two-part structured questionnaire that was partly self-administered and partly filled by the principal investigator or research assistant. See appendix

Socio demographic data was filled into the first part of the questionnaires by the patient or next of kin, assisted by the principal investigator and/or research assistant.

The clinical and radiological data was filled into the second part of the questionnaire by the principal investigator or research assistant following review of the CT request forms, CT images from the console for the data from coronary CTA scans i.e. raw data images, reconstructed images and final radiological reports.

Review of images took place at the imaging facility where the patient had been imaged. It was done by the radiologist at the facility in conjunction with the principal investigator.

\section{Study Variables}

The study variable included age, gender of the patient, presence of risk factors, presenting complaint/clinical presentation, coronary dominance pattern, type of atherosclerotic plaque, coronary vessels involved by plaques, severity of luminal stenosis and isolated coronary vessel anomaly.

\section{Equipment/Personnel}

The CT scanner used is the Aquilion-1 320 MDCT scanner by Toshiba, which has a high temporal resolution enabling the heart to be imaged in a single heart beat in addition to a reduction in radiation exposure to the subjects scanned.

Qualified and certified employed CT radiology technicians operated the CT scanner and follow scanning protocols for cardiac CT imaging as provided by the machine vendor Toshiba.

\section{Data Management and Statistical Analysis}

Data was collected using structured questionnaires. The data collected was entered the statistical package for social science (SPSS) version 20.0. The data was cleaned for errors, inconsistent, missing and duplicated entries to ensure accuracy and high data quality.

Descriptive statistics was presented using percentages and frequencies for categorical or nominal data while mean, standard deviation, median, minimum and maximum was used for continuous variables to characterize the study population. 
Data on clinic-radiological findings was collected using fourteen questions. Correlation statistical test method was applied to analyze these data.

The data results were presented as text, bars, tables, charts and graphs.

\section{Ethical Considerations}

Coronary CT Angiography employs the use of ionizing radiation and iodinated contrast media.

The following ethical guidelines will be used in line with the Helsinki Declaration (World Medical Association - June 1964).

- The name, religion and racial background of the patient were not documented in this study. Patients were identified by $\mathrm{x}$-ray of file number to safe guard confidentiality.

- Approval to carry out the study was sought from Kenyatta National Hospital/University of Nairobi Ethics and Research Committee (KNH/UON-ERC

- Assess to participants' data in form of CT images on the console was only accessible to the authorized imaging staff, reporting radiologists and the referring clinician in cases that needed discussion of results.

- No additional imaging examination was performed other than the one requested by the referring clinician thereby posing NO additional radiation exposure compared to an individual not participating in the current study.

- An information sheet and explanation was provided to the patient outlining the possible risks associated with adverse contrast reactions as well as radiation exposure effects prior to obtaining consent.

- Patients or the next of kin provided both verbal and written consent.

- The patients did not incur any additional cost.

- No blood sample was collected as it is not indicated in this study

- All patients were managed at the optimal standards as personnel and facilities allowed.

- No victimization or preferential treatment was offered to patients because of participating or refusing to participate in the study.

- A copy of the research finding will be given to the facility and the referring clinicians for implementation of any recommendations.

- The results of the examination were availed to the referring clinician immediately to allow for timely and appropriate management.

- All raw data collected will be destroyed upon completion of the study

\section{Results}

\section{Patients' Age}

The mean ages were 55.2 years (SD 14.8) for the males and 56.8 years (SD 19.2) for the females. The age range was 21-78 years and 24-80 years for males and females respectively.

Most patients were aged between 41-60 years (42.9\%) for the male patients and above 60 years (52.9\%) for the females (Table 1).

\section{Gender}

There were more males 21 (55.3\%) than females 17 (44.7\%) in the study (Figure 1).

\section{Cardiovascular Risk Factors}

As shown in Figure 1 below, more females than males had a history of treatment for hypertension $58.8 \%$.

More males reported follow-up and treatment for DM with the occurrence at $23.8 \%$ in contrast to females who commonly reported hypertension at $58.8 \%$. Overall, hypertension was the commonest comorbid risk factor assessed $n=19$.

A total of 3 participants (14.3\%) gave a history of tobacco use in the form of cigarette smoking and all were males. 


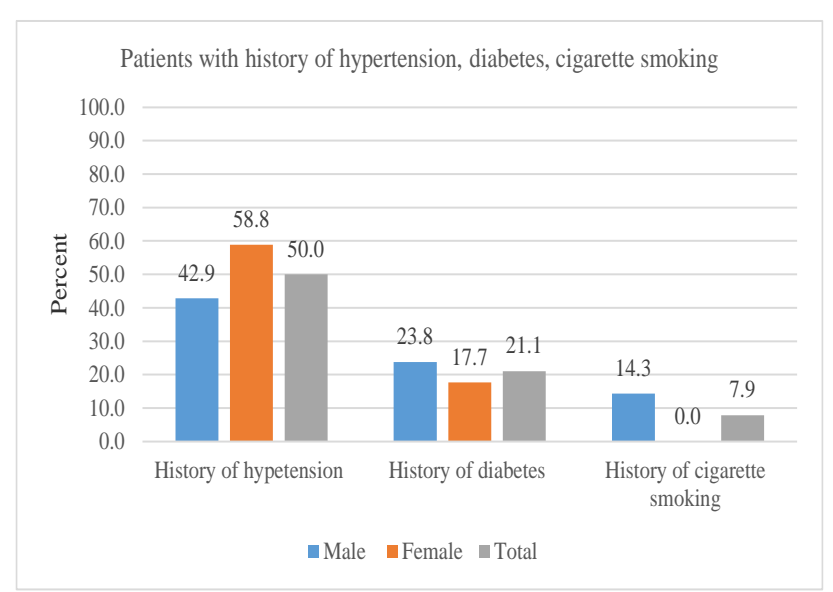

Figure 1. Patients with history of hypertension, diabetes mellitus, cigarette smoking

\section{Clinical Indication for Coronary CTA}

Table 2 below presents the clinical indications noted on the request forms for the patients referred for coronary CTA. Majority of the patients imaged 24 (63.2\%)) presented with chest pain commonly described as angina.

This was followed by an abnormal ECG/ ECHO finding at $42.1 \%$.

Most females had comorbid risk status as an indication for the imaging examinations accounting for $58.8 \%$ compared to the males at $23.8 \%$.
Table 2. Indications for referral for coronary CTA imaging in the study population

\begin{tabular}{|c|c|c|c|c|}
\hline & Male & Female & Total & (n) \\
\hline \multicolumn{5}{|l|}{ Clinical indication (\%) } \\
\hline Chest pain/ angina & 71.4 & 52.9 & 63.2 & 24 \\
\hline $\begin{array}{l}\text { Previous MI (cardiac } \\
\text { arrest) }\end{array}$ & 9.5 & 11.8 & 10.5 & 4 \\
\hline Heart failure & 28.6 & 29.4 & 29.0 & 11 \\
\hline $\begin{array}{c}\text { Abnormal stress ECG/ } \\
\text { ECHO }\end{array}$ & 47.6 & 35.3 & 42.1 & 16 \\
\hline Post stent & 0.0 & 0.0 & 0.0 & 0 \\
\hline Post grafting & 0.0 & 5.9 & 2.6 & 1 \\
\hline $\begin{array}{l}\text { Risk factors - DM, HTN, } \\
\text { renal disease } \\
\text { Pre-cardiac }\end{array}$ & 23.8 & 58.8 & 39.5 & 15 \\
\hline $\begin{array}{c}\text { catheterization/ cardiac } \\
\text { surgery }\end{array}$ & 4.8 & 11.8 & 7.9 & 3 \\
\hline
\end{tabular}

DM, Diabetes Mellitus; ECG, Electrocardiography; ECHO,

Echocardiography; HTN, Hypertension

There was no patient on follow up for post-stenting examination with only $2.6 \% \quad(n=1)$ patient undergoing imaging as a follow up for post grafting.

\section{Association between Age and Clinical Indications}

Majority of the patients with chest pain and/or heart failure as the main clinical indication were older than 61 years $(n=11)$ though there was no statistical significance between the age of the patients and clinical indications for the coronary CTA ( $\mathrm{P}=0.7900$ and 0.1140$)$ respectively (Table $3)$.

Table 3. Association between age and clinical indication for imaging

\begin{tabular}{|c|c|c|c|c|c|c|c|}
\hline & & $0-20$ & $21-40$ & $41-60$ & $61+$ & Total & Fisher's exact test(Pr) \\
\hline \multicolumn{8}{|l|}{ Clinical indication } \\
\hline \multirow[t]{3}{*}{ Chest pain/ anginal pain } & Yes & 0 & 4 & 9 & 11 & 24 & \\
\hline & No & 0 & 2 & 5 & 6 & 14 & \\
\hline & Total & 0 & 6 & 14 & 17 & 38 & 0.7900 \\
\hline \multirow[t]{3}{*}{ Heart failure } & Yes & 0 & 0 & 3 & 8 & 11 & \\
\hline & No & 0 & 6 & 11 & 9 & 27 & \\
\hline & Total & 0 & 6 & 14 & 17 & 38 & 0.1140 \\
\hline \multirow[t]{3}{*}{ Abnormal ECG/ ECHO } & Yes & 0 & 3 & 7 & 6 & 16 & \\
\hline & No & 0 & 3 & 7 & 11 & 22 & \\
\hline & Total & 0 & 6 & 14 & 17 & 38 & 0.7700 \\
\hline \multirow{3}{*}{ Risk factors (Co-morbid status) - DM, HTN, Renal disease } & Yes & 0 & 1 & 6 & 8 & 15 & \\
\hline & No & 0 & 5 & 8 & 9 & 23 & \\
\hline & Total & 0 & 6 & 14 & 17 & 38 & 0.6190 \\
\hline
\end{tabular}

DM, Diabetes Mellitus; ECG, Electrocardiography; HTN, Hypertension 


\section{Association between Gender and Clinical Indication}

More males than females underwent coronary CT angiography for the clinical indications of either chest pain $n=15$, heart failure $n=6$ and/or abnormal ECG/Echo $n=10$ out of a total of 24, 11 and 16 respectively in each category.

However more of the females had comorbid risk factors of diabetes mellitus, hypertension and renal disease combined with a female to male ratio of 2:1 (Table 6).

No significance was however found between the gender of the patient and the clinical indications for the coronary CTA with Fisher's exact test giving $\mathrm{Pi}=0.318,1.000,0.521$ and 0.6190 for chest pain, heart failure, abnormal ECG/Echo and comorbid risk factors

\section{Coronary Dominance Pattern}

Most of the patients in the study demonstrated a right coronary dominance pattern $n=27$ (71.1\%). This was followed by the co-dominant pattern of coronary arteries at $\mathrm{n}=6(15.8 \%)$ and the left dominance pattern being the least common at $n=5$ (13.2\%) (Table 7)

Females had a higher occurrence of right sided dominance with none of the imaged females with a co-dominant pattern of vessel distribution (Figure 2).

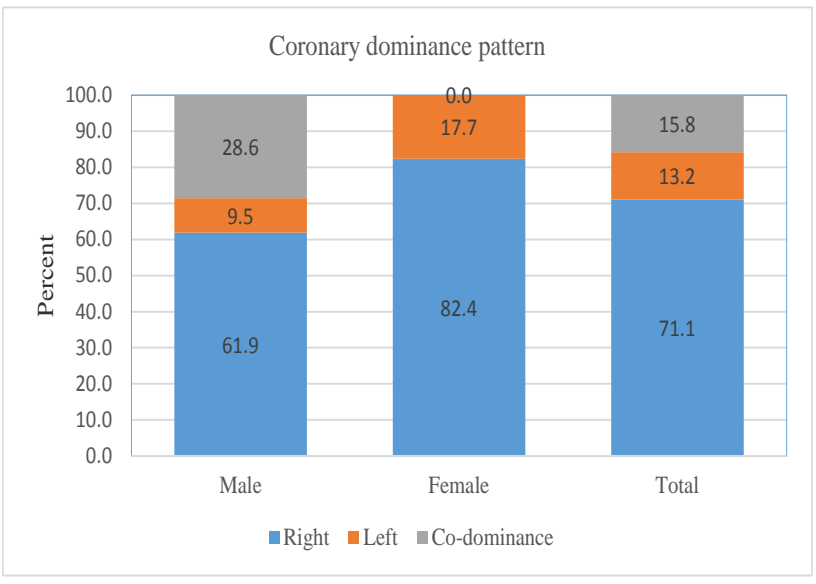

Figure 2. Coronary dominance pattern occurrence in the study population by gender

Table 4. Occurrence and severity of coronary artery imaging findings of the study population

\begin{tabular}{|c|c|c|c|c|}
\hline & Male & Female & Total & (n) \\
\hline \multicolumn{5}{|l|}{ Coronary dominance pattern (\%) } \\
\hline Right & 61.9 & 82.4 & 71.1 & 27 \\
\hline Left & 9.5 & 17.7 & 13.2 & 5 \\
\hline Co-dominance & 28.6 & 0.0 & 15.8 & 6 \\
\hline Presence of coronary atherosclerotic plaques (\%) & 76.2 & 76.5 & 76.3 & 29 \\
\hline \multicolumn{5}{|l|}{ Type of atherosclerotic plaques (\%) } \\
\hline Non-calcified <130 HU & 50.0 & 61.5 & 55.2 & 16 \\
\hline Mixed & 50.0 & 38.5 & 44.8 & 13 \\
\hline \multicolumn{5}{|l|}{ Distribution of coronary atherosclerotic plaques (\%) } \\
\hline RCA & 56.3 & 30.8 & 44.8 & 13 \\
\hline LM & 18.8 & 15.4 & 17.2 & 5 \\
\hline LAD & 81.3 & 69.2 & 75.9 & 22 \\
\hline LCX & 43.8 & 58.3 & 50.0 & 14 \\
\hline \multicolumn{5}{|l|}{ Effect of atherosclerotic plaque (vessel lumen stenosis) } \\
\hline None & 18.8 & 23.1 & 20.7 & 6 \\
\hline Mild 25-50\%\% & 25.0 & 30.8 & 27.6 & 8 \\
\hline Moderate 51-74\% & 31.3 & 30.8 & 31.0 & 9 \\
\hline Severe $>75 \%$ & 25.0 & 15.4 & 20.7 & 6 \\
\hline
\end{tabular}

HU, Hounsfield Units; LAD, Left Anterior Descending artery; LCX, Left circumflex artery; LM, Left main artery; RCA, Right coronary artery 


\section{Abnormal Findings on Coronary CT Angiography}

Out of the total number of imaged patients $n=38,76 \%$ $(n=29)$ had an abnormal imaging finding of either coronary atherosclerotic plaques or aberrant coronary vessel while $24 \%$ $(\mathrm{n}=9)$ had normal coronary and cardiac findings.7)

\section{Distribution of Coronary Atherosclerotic Plaques}

Most of the atherosclerotic plaques were identified and demonstrated on the LAD with an occurrence of $75.9 \%$ $(n=22)$ and least noted on the LM at $17.2 \%(n=5)$.

The females had more of LCX involvement $58.3 \%$ relative to males while the males showed a tendency towards LAD involvement at $81.3 \%$. Overall LAD was predominantly atherosclerotic in both males and females (Figure 3)

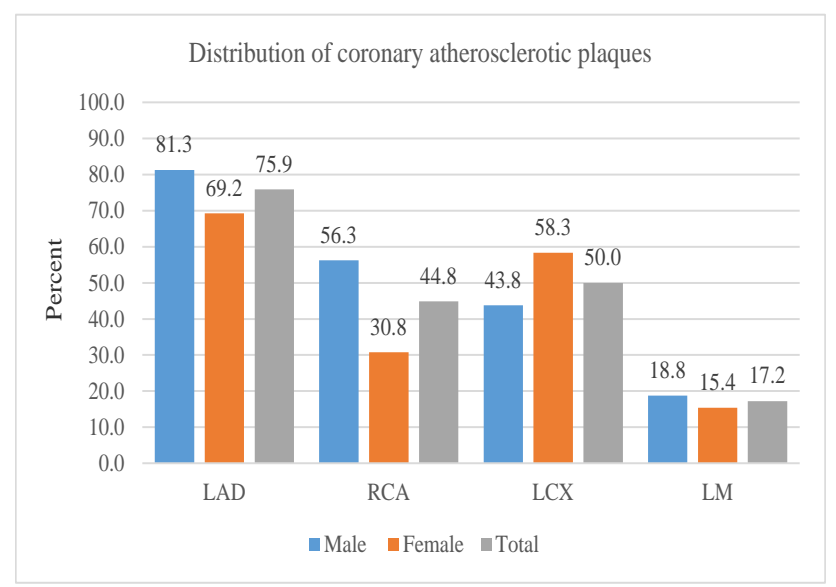

Figure 3. Distribution of coronary atherosclerotic plaques by vessel and gender

\section{Atherosclerotic Plaque Characterization}

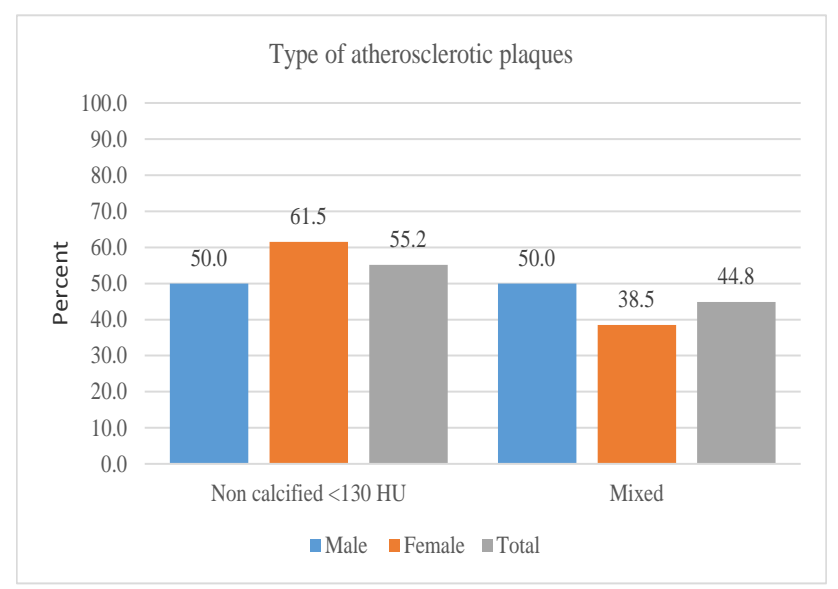

Figure 4. Distribution of atherosclerotic plaque types by gender among the imaged population

Out of a total of 29 patients with positive findings of atherosclerotic plaques, a majority had non-calcified plaques $n=16(55.2 \%)$. A minority $13(44.8 \%)$ had mixed plaques which included calcified, fibrotic and atheromatous plaques occurring together (Figure 4).

The ratio of males with non-calcified to those with mixed plaques was 1:1 while the females tended towards non-calcified plaques with a ratio of 1.5:1 for non-calcified to calcified plaques.

\section{Calcified Plaque Distribution}

Among the study population, a total of 16 patients had detectable and visualized calcified atherosclerotic plaques. (Figure 4 above)

The vessels with calcified plaques overall in descending order were LAD $43.7 \%$, LCX $37.5 \%$, RCA $12.5 \%$ and LM $6.25 \%$. (Figure 5)

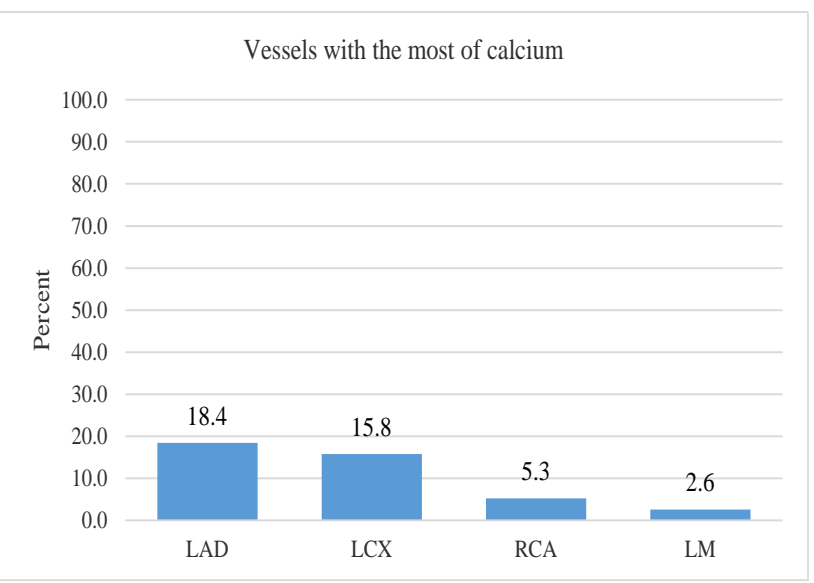

Figure 5. Distribution of calcified atherosclerotic plaques by vessel

The tertiary branches did not demonstrate calcified plaques.

Majority of the females $29.4 \%$ had calcified plaques of the LAD while most males $19.0 \%$ had the calcified plaques on the LCX. None of the males had calcified plaques of the left main coronary artery.

Table 5. Distribution of calcified plaque by vessel type and gender

\begin{tabular}{|c|ccc|c|}
\hline & Male & $\begin{array}{c}\text { Femal } \\
\mathrm{e}\end{array}$ & $\begin{array}{c}\text { Total } \\
\%\end{array}$ & (n) \\
\hline Vessel & & & & \\
RCA & 4.8 & 5.9 & 5.3 & 2 \\
LAD & 9.5 & 29.4 & 18.4 & 7 \\
LCX & 19.0 & 11.8 & 15.8 & 6 \\
LM & 0.0 & 5.9 & 2.6 & 1 \\
\hline
\end{tabular}

\section{Total Calcium Scores (Agatston)}

The highest Agatston total calcium score was 242 and the lowest 0 (zero).

Table 6 below presents the calcium score distribution of the studied population. 
Table 6. Calcified atherosclerotic plaques range as quantified by Agatston score among the imaged patients population

\begin{tabular}{|c|ccc|c|}
\hline & Male & Female & Total & $(\mathrm{n}=16)$ \\
\hline Calcium levels & & & & \\
Min & 0.0 & 0.0 & 0.0 & \\
Max & 79.0 & 242.0 & 242.0 & \\
Mean & 4.9 & 26.3 & 14.5 & \\
SD & 17.1 & 61.4 & 43.6 & \\
\hline
\end{tabular}

\section{Atherosclerotic Plaque Effect on Coronary Artery Lumen}

Majority of the study subjects had their coronary vessel lumens either mildly or moderately stenosed by atherosclerotic plaques representing 58.6\% ( $\mathrm{n}=17)$. The proportion of individual with coronary vessels demonstrating severe luminal narrowing i.e. $>75 \%$ stenosis to those with insignificant vessel stenosis $<25 \%$ stenosis was 1:1 (Table 7)

Table 7. Effect of atherosclerotic plaque on vessel lumen (stenosis level)

\begin{tabular}{|c|ccc|c|}
\hline & Male & Female & Total & (n) \\
\hline $\begin{array}{c}\text { Effect of atherosclerotic plaque } \\
\text { (vessel lumen stenosis) }\end{array}$ & & & & \\
None & 18.8 & 23.1 & 20.7 & 6 \\
Mild 25-50\%\% & 25.0 & 30.8 & 27.6 & 8 \\
Moderate 51-74\% & 31.3 & 30.8 & 31.0 & 9 \\
Severe $>75 \%$ & 25.0 & 15.4 & 20.7 & 6 \\
\hline
\end{tabular}

\section{Associated Cardiac Findings}

Cardiac findings of significance during the scanning were cardiomegaly and pericardial sac calcifications. Cardiomegaly was gauged as a cardiothoracic ratio of more than 0.5 with measurements taken at the widest cardiac and thoracic wall dimensions. Pericardial calcification was identified on the calcium score scan and automatically detected by the Vitrea software.

As represented in table 8 below, majority of the imaged population with cardiac findings had cardiomegaly this representing $(n=13) 34.2 \%$ of the imaged persons while a minority had pericardial calcifications representing $(n=3)$ $7.9 \%$.

Majority of the individuals with cardiomegaly as a concurrent finding were females at $52.9 \%$. None of the females had any pericardial calcifications seen exclusively in the males.

Table 82. Associated cardiac findings

\begin{tabular}{|c|ccc|c|}
\hline & Male & Female & Total & (n) \\
\hline Cardiac findings (\%) & & & & \\
Cardiomegaly & 19.1 & 52.9 & 34.2 & 13 \\
Pericardial calcification & 14.3 & 0.0 & 7.9 & 3 \\
\hline
\end{tabular}

Illustration 1: A volume rendered 'vessel only' reconstruction demonstrating an aberrant origin of the RCA with an inter-arterial malignant course between the Right Ventricular Outflow Tract and the aorta. This is a coronary CTA image of a 47-year-old male patient presenting with intermittent angina. There were no significant coronary atherosclerotic plaques of the coronary vessels. This was an isolated finding.

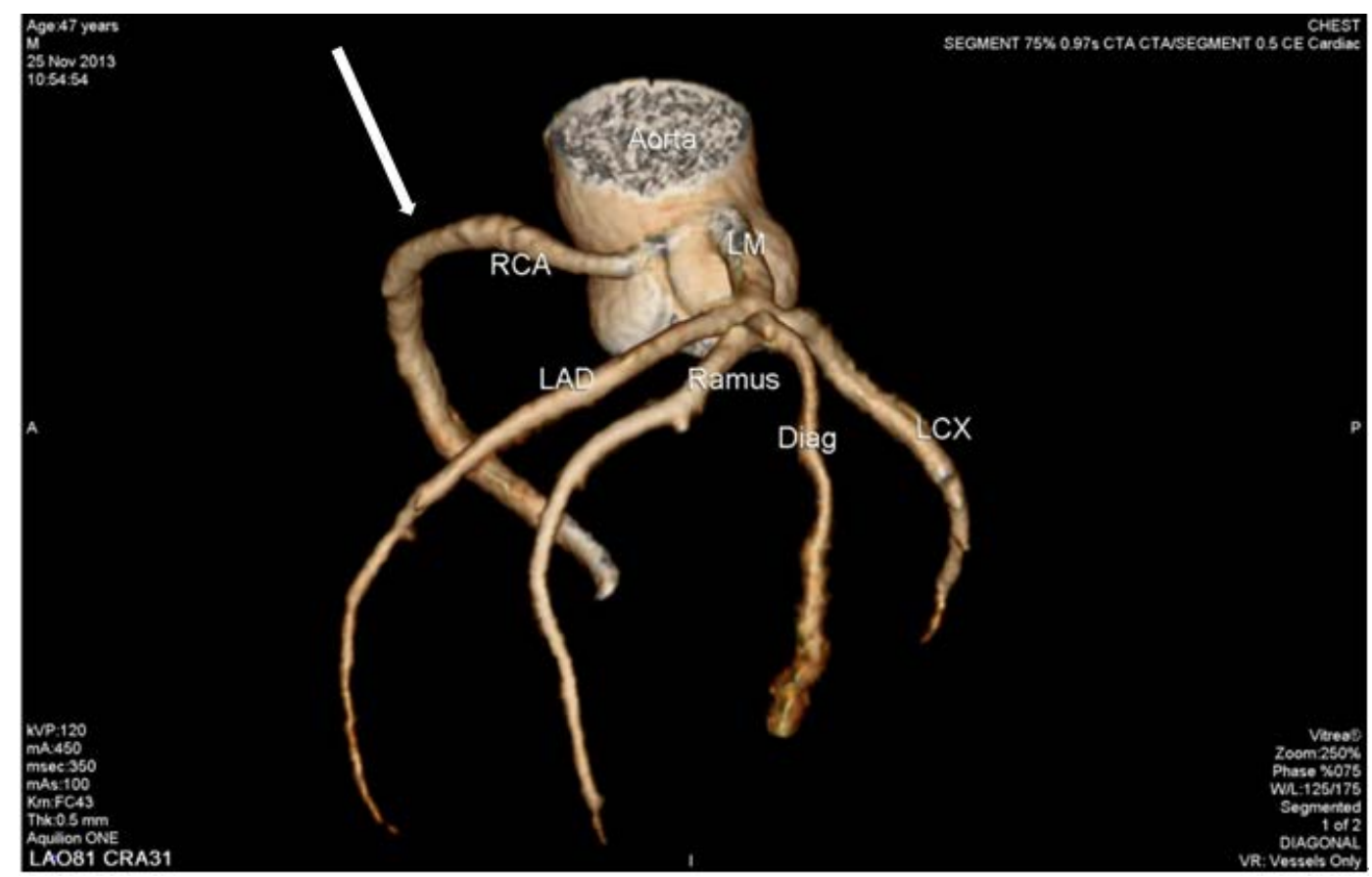


Illustration 2: A volume rendered 'heart and vessels' coronary CTA image illustrating left dominant coronary circulation in a 41-year-old male with an abnormal MPS and a family history of CAD. The coronary CTA findings were normal.

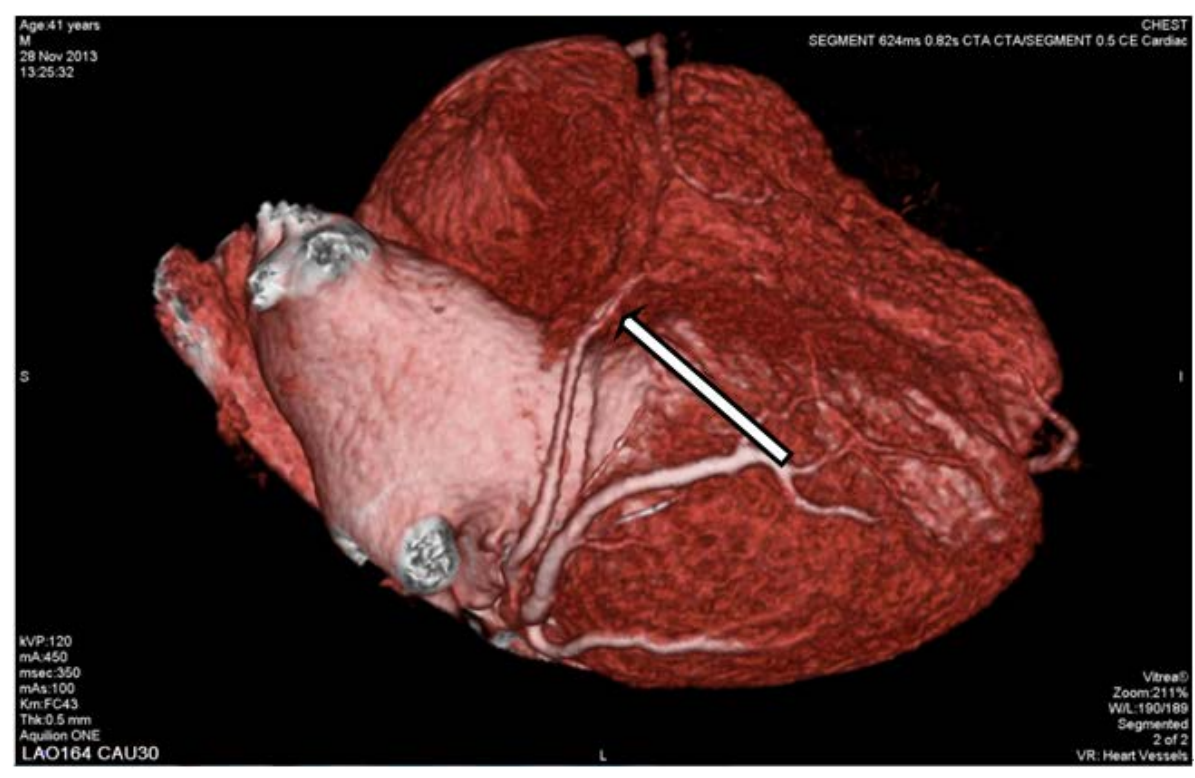

Illustration 3: Volume Rendered Image Illustrating a Right Dominant Coronary Circulation. A 54-year-old female presenting with chest pain and a history of hypertension

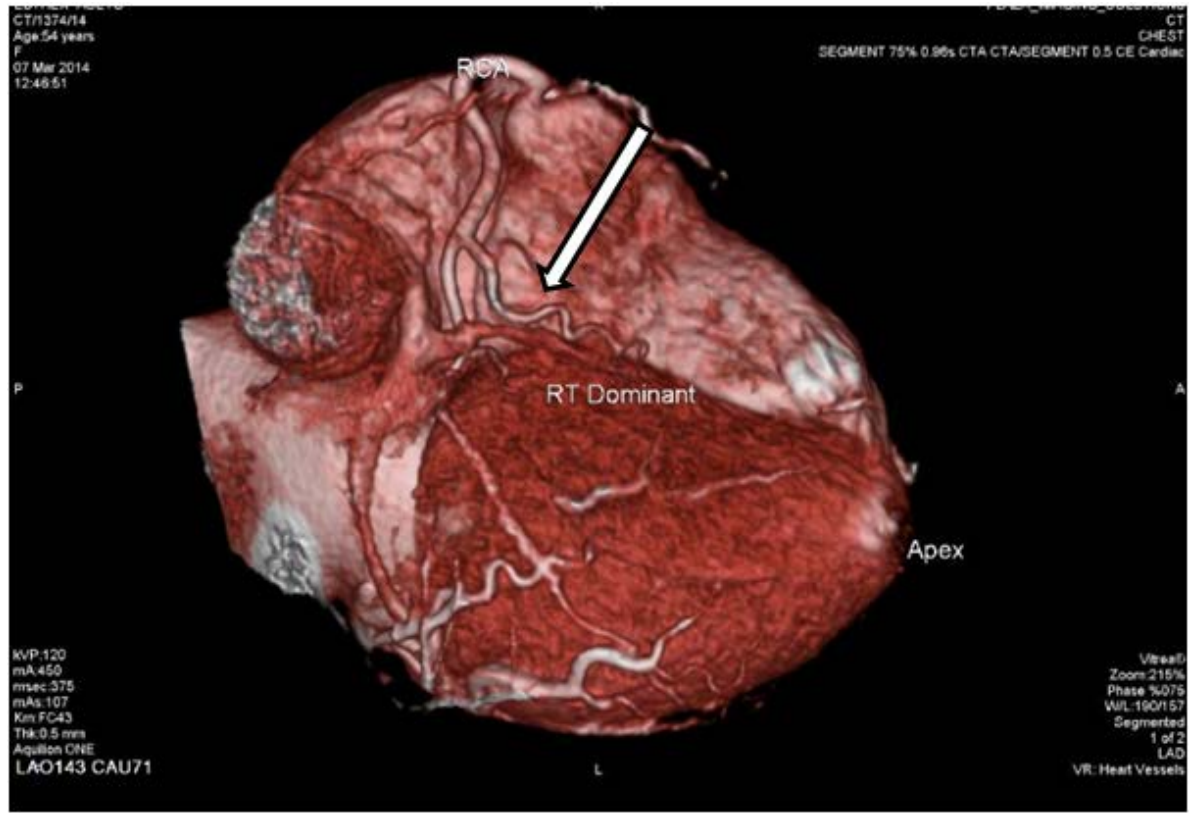

Illustration 4: Volume Rendered 'Transparent Heart' Image Illustrating an aneurysm of the LM coronary artery. A 52-year-old male with a recent MI persistent chest pain 


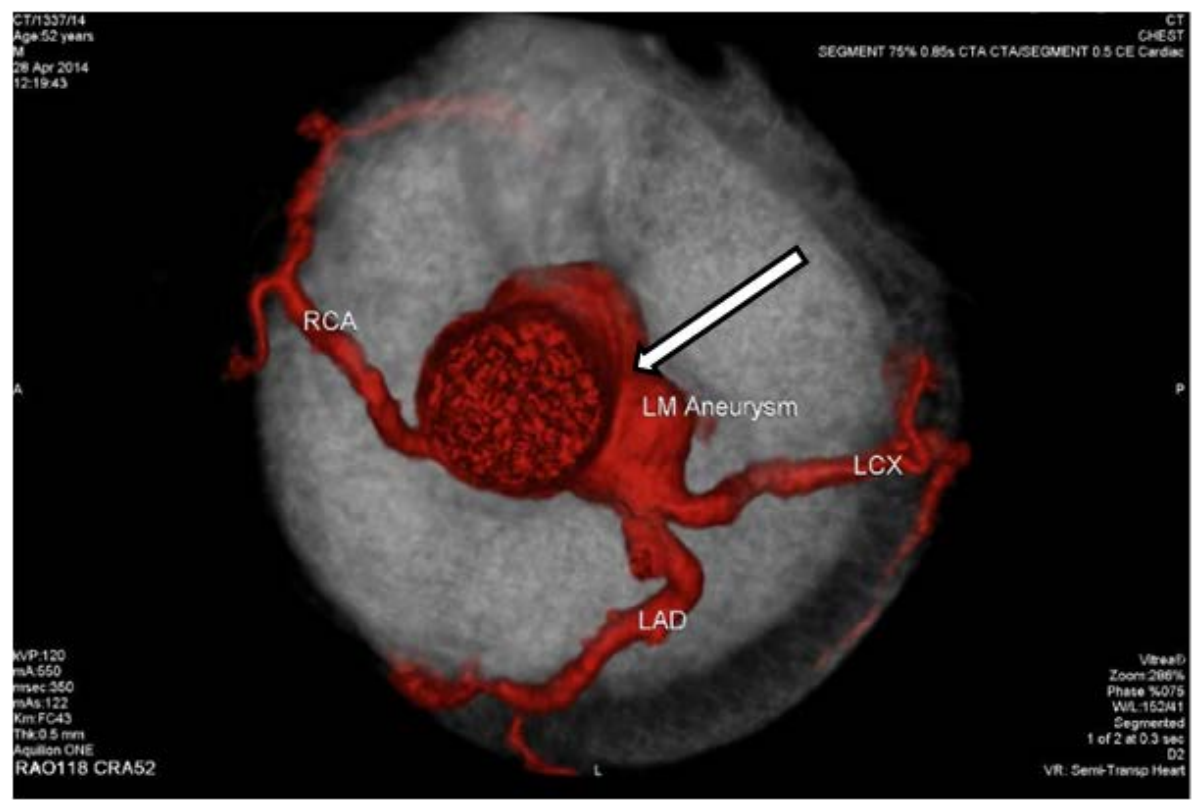

Illustration 5: An Image of the different types of atherosclerotic plaques causing luminal stenosis. A maximum intensity projection (MIP) curved planar reformat of a 74-year-old with long standing intermittent chest pain and history of myocardial infarction

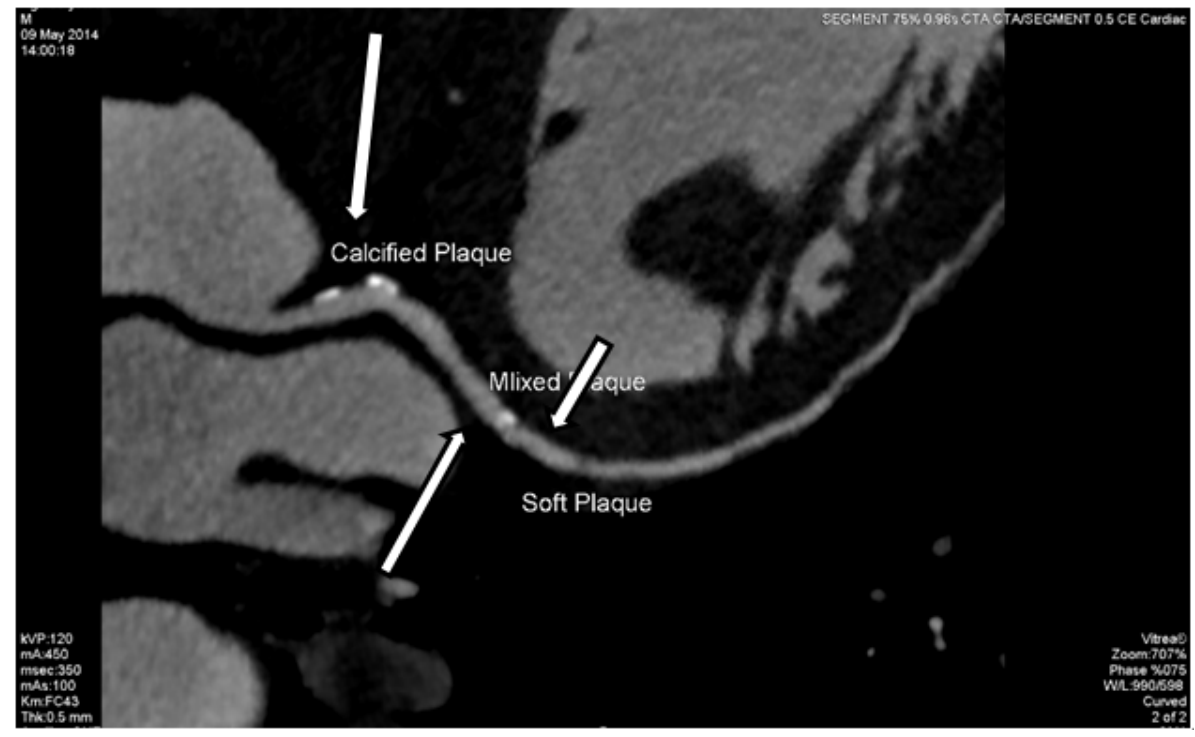

Illustration 6: Volume rendered CTA demonstrating absence of the left main coronary artery with direct origin from the left coronary sinus of the LAD, Ramus Intermedius and LCX 


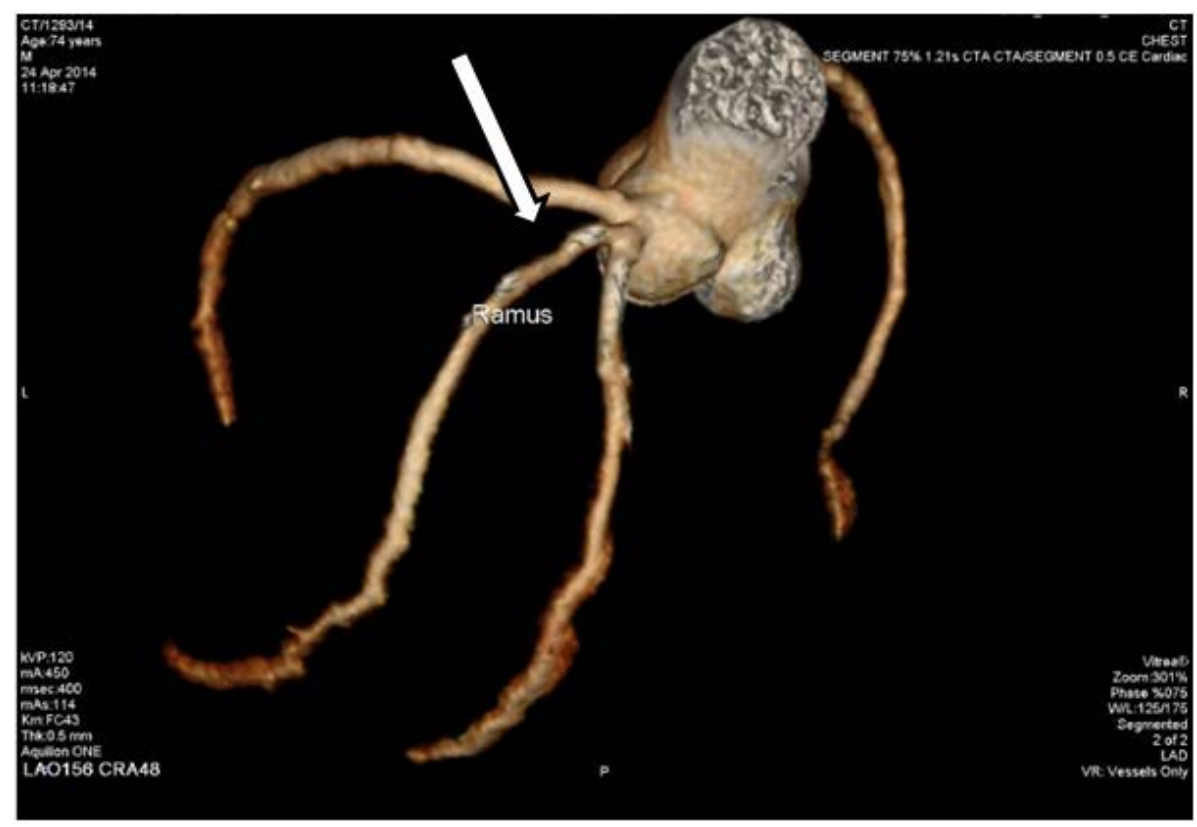

\section{Discussion}

Coronary artery disease is the overall leading cause of mortality and morbidity worldwide with a persistent rise in prevalence seen over the decades. A similar trend has been observed in the developing world though data to this effect is lacking (4, 5, 6). Coronary CTA performed using CT scanners of minimum 64 slice has recently become a one-stop shop for investigation of chest pain in patients suspected for coronary artery disease $(16,17)$. The main objective of this study was to determine the spectrum and distribution of imaging findings at coronary CTA in patients suspected to have coronary artery disease.

The current study shows that the age range of patients undergoing coronary CT angiography is wide (range, 21-80 years) with a mean age of 56 years. Majority of the patients imaged were aged 61 years and above. These findings compare with a previous conclusion by Kelly et al. (30) and other studies $(31,32)$ which showed relatively similar findings with a mean age of 56 years and $89 \%$ of the imaged patients being over the age of 45 years. This illustrates the known fact that the likelihood of coronary artery disease increases with advancing age and there is a high probability of an older individual undergoing coronary CTA.

Gender difference was observed in the study with a male predominance of $55.3 \%$ compared to females at $44.7 \%$. Though the figures vary slightly, other studies reveal a similar proportion of the genders $(30,31,32)$ with the females showing a consistently lower likelihood of being referred and undergoing imaging compared to the males. These findings may be explained by the predilection of males to acquire coronary artery disease at an earlier age with women only matching the occurrence after the onset of menopause and more so in the postmenopausal period. This observation follows the widely known protective role of endogenous estrogen on the cardiovascular system in the reproductive years of the females (33). The difference between the current study with a female rate of $44.7 \%$ compared to the study by Kelly et al. (30) may also be explained by the fact that delay in health seeking in the local population is predominantly exhibited by males.

Comorbid state of hypertension was common among the imaged subjects in the current study at 50\% which greatly surpassed diabetes mellitus at $21 \%$. This current finding is comparable to other studies $(31,32$,) which showed most CAD patients to suffer concurrently from hypertension defined as persistently elevated blood pressure of more than 140 systolic and 90 diastolic measurements taken correctly in more than two instances in persons under 80 years of age (34). The studies probed the role of risk factors on plaque morphology and concluded that hypertension is the single most common independent risk factor for developing accelerated coronary atherosclerotic disease. The high incidence of hypertension in patients undergoing coronary CTA likely demonstrates the strong pathophysiological role of elevated blood pressure on atherosclerotic plaque initiation, progression and development which may in effect accelerate coronary atherosclerotic disease. This therefore puts patients with hypertension at a higher risk for CAD in addition to hypertensive heart disease (35).

Comparatively, diabetes mellitus type II was not as prevalent in this group of patient population undergoing coronary CTA. This is paradoxical given the fact that diabetes mellitus type II has been reclassified as a coronary risk equivalent in the Framingham risk score for individuals with CAD as opposed to a risk factor $(36,37)$. A CAD risk equivalent is defined as a condition that places an individual at a higher risk for experiencing a cardiac 'hard event' or even equivalent to an individual who has already 
experienced a hard event (38) Studies done by other researchers reflect a similar trend $(30,31)$. This paradox is probably explained by the findings of a study by Saely et al. (39) which did conclude that diabetes mellitus type II per se is not a risk equivalent for CAD as is perceived in many epidemiological studies (36).

Cigarette smoking was associated though not proven in the current study with a higher occurrence of calcified atherosclerotic plaques which has been studied and documented as a true marker of high atherosclerotic plaque burden and therefore CAD. Active current cigarrete smoking is known to worsen the acute coronary symptoms due to the endothelial dysfunction induced by tobacco causing disordered nitric oxide vasodilatory effect on the vasculature $(35,40)$. In the current study cigarette smoking accounted for a minority of the imaged subjects and was only seen in the males.

Regarding coronary dominance pattern, the study reproduces the well-known findings of various studies which have shown a large majority of individuals to have a right dominant coronary pattern and the least numbers to be left dominant with the balanced/ co-dominance pattern being slightly more than the left dominant (23). A unique finding in the current study is the co-dominance pattern exclusively observed in the male patients.

The current study findings demonstrate a high incidence of coronary atherosclerotic plaques in majority of the imaged patients. Most of these patients had non-calcified plaques. The atherosclerotic plaques were shown to be inequitably distributed with most of the plaques found on the walls of the proximal LAD. The spatial distribution of majority of the plaques correlates with the geometry of vessels and their hemodynamics including wall shear pressure that is observed particularly at the proximal LAD. These findings correlate with other studies of atherosclerotic plaque distribution which concluded that atherosclerotic plaques tend to cluster within the proximal third of vessels leading to predictable 'hotspots' for vessel occlusion (41). Flow patterns have been studied and found to affect initiation and development of plaques (42). A study by Svindland on histological coronary vessels analysis concluded that the left anterior descending artery (LAD) had the earliest and most prominent fibrous and sudanophilic plaque appearance (43). More females than males showed a higher likelihood of non-calcified plaques but the difference of plaque type in relation to gender was not significant.

Both LAD and LCX showed a higher propensity for calcified plaques compared to RCA and LM with there being a significant association between calcified plaque burden and vessel. As noted in previous studies, calcified plaques are a direct reflection of overall plaque burden and therefore risks determinant for coronary 'hard events'. This likely explains the initial presentation of myocardial infarction or even sudden death in unsuspecting individuals with CAD as LAD known colloquially as 'the widow maker' is the single most affected vessel by atherosclerotic plaques as evidenced by the study.

Multi-vessel involvement by atherosclerotic plaques was observed though not analyzed, with most patients demonstrating at least two-vessel disease.

Most plaques caused mild to moderate vessel lumen stenosis (58.6\%) as calculated by luminal diameters versus vessel wall diameters and luminal cross-sectional area of areas with identifiable plaques using the Vitrea workstation in- built software. This explains the fact that most patients presented with intermittent anginal chest pain (63.2\%) as opposed to previous myocardial infarction (10.5\%) which is likely to be caused by a more severe vessel lumen stenosis.

Cardiomegaly was a common associated finding being seen in up to $52.9 \%$ of females and less commonly in males. This finding correlates well with the comorbid status of hypertension seen in majority of the females in the study compared to men. Hypertension as is known leads to elevated systemic blood pressure which exerts a pressure load on the left side of the heart resulting in myocardial hypertrophy appreciated as cardiomegaly on imaging.

\section{Conclusions}

The study concludes that there is an overall high incidence of atherosclerotic cardiovascular disease in the local middle age and elderly African population with no gender predilection seen after the menopausal period.

Coronary symptoms prompting investigation are notably common amongst persons suffering from concurrent hypertension.

Multi-vessel atherosclerotic disease is common in patients with mild and moderate luminal narrowing within the walls of LAD and RCA with most plaques being non-calcified.

Calcified atherosclerotic plaque were rare amongst the study population even in patients demonstrating significant stenosis from plaque luminal occlusion leading to a conclusion that in the local black African population, calcified plaques quantification by MDCT calcium score scan may not accurately predict the atherosclerotic plaque burden accurately. Therefore, direct visualization and characterization without a calcium scan may suffice and be sensitive in classifying patients undergoing coronary CTA for suspected CAD. This is in addition to reducing the radiation exposure of patients undergoing coronary CTA.

Coronary calcium scoring is not essential in patients of African descent as the study elucidated no correlation between total calcium score and the atherosclerotic burden of disease

Isolated anomalous coronary vessel origin is rare, with specific individual unique vessel arrangement and branching pattern noted of the distal left coronary artery branches. The proximal main vessels pattern was generally replicated amongst the studied subjects. 


\section{Recommendations}

Concerning patient selection for coronary CTA, there is need for classifying suspected or confirmed individuals with CAD into low, intermediate and elevated risk to maximally derive benefit from the imaging options offered and subsequent management. This could be achieved by use of the Framingham scoring system.

Based on the above findings and conclusion, cardiac CTA is indicated in persons over the age of 55 years with symptoms of ischemic heart disease.

\section{Declaration}

I, Dr. Eunice Akinyi Omamo, declare that the work therein contained is my original idea and has not been submitted or presented in any forum/place to the best of my knowledge.

\section{Acknowledgements}

I am grateful to my supervisors Dr. Alfred Odhiambo and Dr. Fredrick Bukachi for their guidance, advice and support throughout the entire study period.

Thank you to all the technical and support staff at Plaza Imaging Solutions including the radiographers, typists and all other staff for the cooperation accorded to me during the undertaking of the study and especially for assistance in keeping track of the recruited patients during the data collection period, for retrieval of reports and for patient follow up.

Thank you to the respondents recruited into the study and their kin for their immense cooperation during the study.

The statistical assistance offered by Mr. Felix Mulama cannot be overstated, am grateful.

Finally, my heart felt gratitude goes to my immediate family members and especially to my husband Bernard Olayo for script management and my children for their understanding during the entire period.

\section{Dedication}

This dissertation is dedicated to my dear husband Dr. Bernard Olayo and to my beloved children Andrew, Elena and Adrian.

\section{Abbreviations}

\begin{tabular}{|c|c|}
\hline CAC & Coronary Artery Calcium \\
\hline CAD & Coronary Artery Disease \\
\hline CC & Coronary Calcium \\
\hline CHD & Coronary Heart Disease \\
\hline CT & Computed Tomography. \\
\hline ССТА & Coronary Computed Tomography \\
\hline \multicolumn{2}{|l|}{ Angiogram } \\
\hline $\mathrm{DM}$ & Diabetes Mellitus \\
\hline EBCT & Electron Beam \\
\hline \multicolumn{2}{|l|}{ Tomography } \\
\hline ECG & Electrocardiography \\
\hline FRS & Framingham Risk Score \\
\hline FS & Framingham Study \\
\hline IHD & Ischemic Heart Disease \\
\hline LAD & Left Anterior Descending \\
\hline \multicolumn{2}{|c|}{ Coronary Artery } \\
\hline LDH & Low Density Lipoproteins \\
\hline LM & Left Main Coronary Artery \\
\hline LCX & Left Circumflex Coronary Artery \\
\hline MDCT & Multi Row Detector Computed \\
\hline \multicolumn{2}{|l|}{ Tomography } \\
\hline MI & Myocardial Infarction \\
\hline MPS & Myocardial Perfusion Scanning \\
\hline NPV & Negative Predictive Value \\
\hline PPV & Positive Predictive Value \\
\hline RCA & Right Coronary Artery \\
\hline TC-99M & Technetium $99 \quad$ Metastable \\
\hline
\end{tabular}




\section{Appendix I:}

Estimated budget

\begin{tabular}{|l|l|c|}
\hline Allocation & Break Down & Amount in Kes. \\
\hline Stationary & 2 reams Printing paper@ 1000/- & 2,000 \\
\hline Biro pens 10 pieces @ 30/- & 300 \\
\hline 5 Folders @200 & Ethics Fee & 1,000 \\
\hline Ethics board & Typist fees & 4,000 \\
\hline Secretarial services & & 5,000 \\
\hline Photocopy and binding & Laptop Computer & 3,000 \\
\hline Computer and Printer & 65,000 \\
\hline Computer soft wares & 6,000 \\
\hline Printer and Cartridges & 8,000 \\
\hline Internet hours & & 4,000 \\
\hline Data collection and analysis & 50 hours@ 80/hr. & 5,000 \\
\hline Research assistants & Statistician services & 20,000 \\
\hline Printing and Binding & & 2,000 \\
\hline Final report & Proposal & 8,000 \\
\hline Contingencies & & 15,330 \\
\hline Total Amount & Contingencies (10\%) & $\mathbf{1 6 8 , 6 3 0}$ \\
\hline
\end{tabular}

\section{Appendix II: Consent Explanation Form}

My name is Dr. Omamo Eunice Akinyi, a Master of Medicine in Radiology student in the department of Diagnostic Imaging and Radiation Medicine at the University of Nairobi. I am conducting a study titled „The spectrum of imaging findings of coronary CT angiography in patients suspected to have coronary artery disease ${ }^{\text {ee }}$ with approval of the KNH/UON-ERC.

The purpose of the study is to find out the age and gender distribution of patients referred for coronary CT angiography as well as the occurrence and severity of coronary artery disease in the imaged population. It also aims to correlate the clinical presentation with the imaging findings.

Coronary CT angiography will be performed using a CT scanner which utilizes ionizing radiation to form images of the internal organs including the coronary arteries which are made conspicuous by use of intravenous iodinated contrast media.

Your attending doctor referred you for the coronary CT angiography examination to help him/her in the further management of your cardiac condition.

There is a possibility of an adverse reaction with the contrast media use and a very remote possibility of radiation induced carcinogenesis from exposure to the x-rays. It is not predicted that you will suffer any harm by participating in this study

However, this examination is very beneficial to you as it will inform and assist your primary physician/ cardiologist to better manage your cardiac condition and make important decisions regarding your current and future treatment options by providing some diagnoses, answers and insight about your coronary arterial tree. Furthermore, the Aquilion $1 \mathrm{CT}$ scanner used for the examination is specially designed to significantly reduce the amount of radiation exposure during scanning thus reducing the risks associated with high radiation exposures.

I would like you/your kin to be a part of this study.

Please note that your participation is voluntary, you have a right to decline or withdraw from the study at any time. You will not incur any extra costs by participating in the study. The research study does not pose any additional danger to you neither is it additional to that requested examination by your doctor.

Should you agree to participate, you will be required to fill in a short questionnaire detailing your bio-data.

The findings obtained from this study will be kept confidential, your name will not be used or appear anywhere. Only the x-ray number will be used for reference purposes and once the study ends all the information will be destroyed. 30

The results of this study will be used to improve on management of patients with suspected or confirmed coronary artery disease and add to the knowledge base of the practicing physicians.

Should you wish not to participate, you will continue to receive treatment as scheduled and you will not be discriminated against in any way.

In case of issues arising from the study, you can contact me, my supervisors or the $\mathrm{KNH} / \mathrm{UON}$-ERC through the chairman. 


\section{Appendix III: Research Consent Form}

I. having read the consent explanation form and having been explained to, do voluntarily agree to participate in this study titled 'The spectrum of imaging finding of coronary CT angiography in patients suspected to have coronary artery disease at Plaza Imaging Solutions Limited." I am also aware that I can withdraw from this study without losing my healthcare benefits or the quality of management of my condition being compromised.

Signature

Date.

I . certify that the patient/guardian has understood and consented participation in the study.

Signature (Witness)

Date

\section{Appendix IV: Data Collection Form}

\section{Part 1}

Questionnaire No.
1. Age
$0-20$
$21-40$
$41-60$
$>60$

2. Gender

M

F

3. Weight (kg)

4. History of hypertension

YES

NO

UNKNOWN

5. History of diabetes

YES

NO

UNKNOWN

6. History of Cigarette smoking

YES

NO

UNKNOWN

7. Previous cardiac imaging

CTA

CMRI

MPS

STRESS ECG

CCA

\section{Part 2}

1 Clinical indication/summary for the examination as given in the request form

Chest pain/ anginal pain

Previous MI (cardiac arrest)

Heart failure

Abnormal ECG/ Stress test

Post stent

Post grafting

Risk factors (Co-morbid status) - DM, HPTN, Renal disease 
2 Coronary dominance pattern

\begin{tabular}{|c|c|c|c|c|}
\hline RIGHT & \multicolumn{3}{|c|}{ LEFT } & CO-DOMINANCE \\
\hline & 3 & Anomalous & ronary artery(s) origin & \\
\hline \multicolumn{4}{|c|}{ Yes } & No \\
\hline \multicolumn{5}{|c|}{ If yes, which vessel (tick as appropriate) } \\
\hline \multirow[t]{2}{*}{ RCA } & $\mathrm{LM}$ & & LAD & LCX \\
\hline & & Type & origin anomaly & \\
\hline Single origin for both RCA and LM & LM from right & coronary sinus & RCA from left coronary sinus & Coronary vessel from pulmonary trunk \\
\hline
\end{tabular}

\section{Type of Arterial Anomaly}

$1 \quad$ Myocardial bridging

\begin{tabular}{ccccc}
\hline \multicolumn{1}{c}{ Yes } & & No & \\
\hline & & & & \\
& If yes which vessel & & \\
\hline RCA & LM & LAD & LCX & PDA \\
\hline
\end{tabular}

\section{Anomalous Termination/Distal Extent}

3 If yes, which vessel

$\begin{array}{llll}\text { RCA LAD } & \text { LCX }\end{array}$

4 Type of anomalous termination/distal extent

Aneurysmal termination

Intramural termination

5 Presence of coronary atherosclerotic plaques

Yes No

6 Type of atherosclerotic plaques

Non-calcified $<130 \mathrm{HU}$

Calcified $>130 \mathrm{HU}$

Mixed

$7 \quad$ Distribution of coronary atherosclerotic plaques

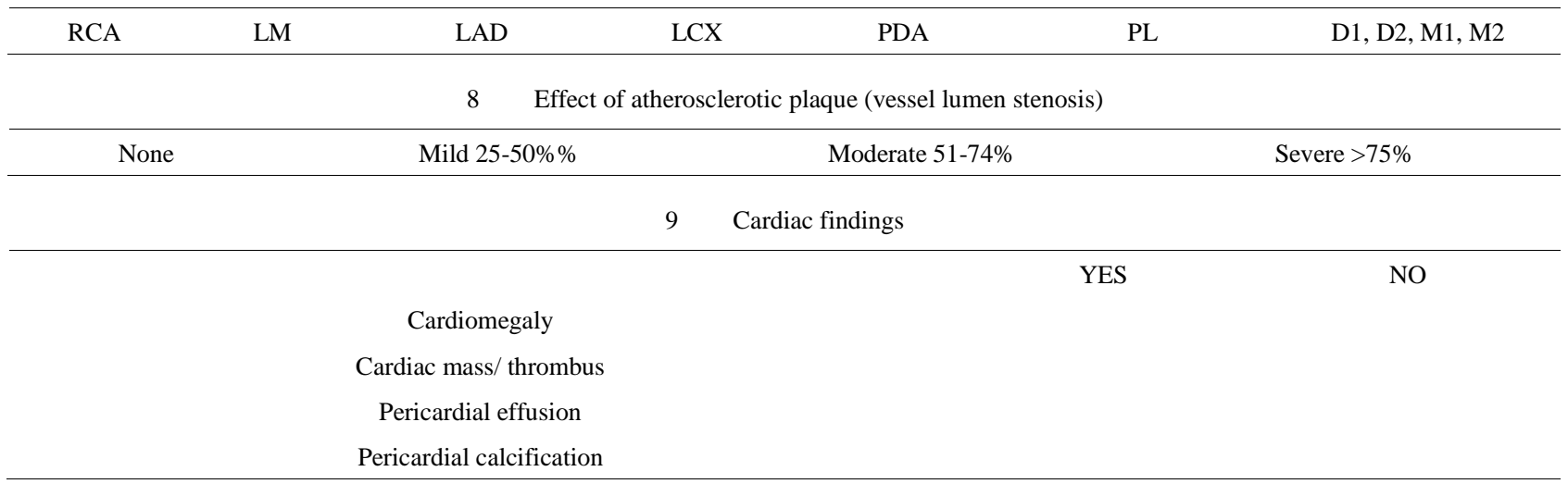




\section{Appendix V: Time-Frame}

The following is a proposed time-frame of the study process:

\begin{tabular}{|c|c|c|}
\hline Number & Activity & Estimated Time \\
\hline 1 & Proposal Development & January- July 2013 \\
\hline 2 & Proposal Submission to the department for marking 2013 \\
\hline 3 & Submission of proposal for ethical approval & September 2013 \\
\hline 4 & Data Collection & January 2013 to May 2014 \\
\hline 5 & Data Analysis 2014 \\
\hline 7 & Dissertation writing & May-June 2014 \\
\hline 8 & Dissertation submission & June 2014 \\
\hline
\end{tabular}

\section{REFERENCES}

[1] Strong J.P, McGill H.C Jr. The natural history of coronary atherosclerosis. Am J Pathol (1962) 40:37-49.

[2] Libby P, Theroux P. Pathophysiology of coronary artery disease. Circulation. Am Heart Assoc (2005).111:3481-3488

[3] Ross R. The pathogenesis of atherosclerosis: A perspective for the 1990s, Nature 362:1993, 801-809

[4] American Heart Association. Heart disease and stroke statistics 2004 update. Dallas Tx; American Heart Association (2003)

[5] Roger, V., Go, A.S, Lloyd-Jones et al (2011). Heart disease and stroke statistics-2012 Update: A report from the American Heart Association. Circulation. Retrieved January 2012, http://circ.ahajournals.org/content/early/2011/12/15/12/CIR.0 b013e31823ac046.DC1.html

[6] O Akinboboye, O Idris, O Akinboboye, O Akinkugbe. Trends in coronary artery disease and associated risk factors in sub Saharan Africans (2003). J Hum Hypertens 17:381-387

[7] Rybicki J.F, Otero J.H, Steigner M. L, Vorobiof G, Nallamshetty L., Mitsouras D. et al. Initial evaluation of coronary images from 320 detector row CT (2008) [published online March 2008] cited July 2013. Int J Cardiovasc Imaging (2008) 24:535-546.

[8] Heart and stroke statistical update. Dallas, Texas. American Heart Association (2001)

[9] O’Rourke R. A et al. “American College of Cardiology/American Heart Association Expert Consensus Document on Electron Beam Computed Tomography for the Diagnosis and Prognosis of Coronary Artery Disease.” J Am Coll Cardiol 36.1(2000): 326-340)

[10] Kenya Factsheets of Health Statistics (2010)

[11] ACCF/AHA 2007 Clinical Expert Consensus Document on Coronary artery calcium scoring by Computed Tomography in Global Cardiovascular Risk Assessment and in evaluation of patients with chest pain. A report of the American College of Cardiology Foundation clinical expert consensus task force (ACCF/AHA Writing committee to update the 2000 Expert
Consensus Document on Electron Beam Computed Tomography). Developed in collaboration with the society of atherosclerosis imaging and prevention and the society of cardiovascular computed tomography.

[12] Okraine, K. Devi K. B., Eisenberg M. J., (abstract) Coronary artery disease in the developing world. Am. Heart J. (2004) 148(1), 7-15

[13] Sangiorgi G, Rumberger J a Severson A et al. Arterial calcification and not lumen stenosis is highly correlated with atherosclerotic plaque burden in humans: A histological study of 723 coronary artery segments using non-decalcifying methodology. J Am coll cardiol.1998; 89: 36-44

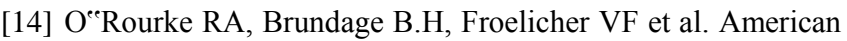
College of Cardiology/ American Heart Association Expert Consensus Document on EBCT for the diagnosis and prognosis of coronary artery disease, J Am Coll Cardiol. Circulation 2000; 102:126-140

[15] Wexler L., Brundage B., and Crouse J., Detrano, R., Fuster, V., Maddahi, J., and Taubert, K. Coronary Artery Calcification: Pathophysiology, Epidemiology, Image Methods and Clinical Implications. A scientific statement from the American Heart Association. Circulation (1996) 94.5, 1175-1192

[16] Gil, Bachar N., Ran, K., Tamar, G., Shmuell F., Eli A. (2007) Prevalence of Significant Non-Cardiac Findings on Coronary Multidetector Computed Tomography Angiography in Asymptomatic Patients. Journal of Computer Assisted Tomography, 31(1), 1-4

[17] Stephen Schroeder, Stephan Achenbach, Frank Bengel et al. Cardiac Computed tomography; indications, applications, limitations and training requirements. Report of a writing group deployed by a working group Nuclear Cardiology and Cardiac CT of the European Society of Cardiology and the European council of Nuclear Cardiology. Eur Heart J (2008) 29:531-556

[18] Kopp. A F, Ohnesorge B., Flohr T., Georg C., Schroder S., Kuttner A. et al. Cardiac Multidetector row CT: First clinical results of retrospectively ECG-gated spiral with optimized temporal and spatial resolution, RöFo; Fortschritte auf dem Gebiete der Röntgenstrahlen und der Nuklearmedizin 2000 May; 172 (5) 429-35

[19] Flohr T.G., CH McCollough et al. First performance evaluation of a dual source CT (DSCT) system, Eur Radiol (2006)16:256-268 
[20] Stary H.C. Composition and classification of human atherosclerotic lesions, Virchows Arch a Pathol Anat Histopathol 4211992 277-290

[21] Budoff, M. J., Dowe D., Jollis J. G., Gitter M., Sutherland J., Halamert E. Et al. Diagnostic Performance of 64 Multidetector Row Coronary Computed Tomographic Angiography for Evaluation of Coronary Stenosis in Individuals Without Known Coronary Artery Disease from the Prospective Multicenter ACCURACY (Assessment of Coronary Computed Tomographic Angiography of Individuals Undergoing Invasive Coronary Angiography) Trial. J Am Coll Cardiol. 2008; 52(21). 1724-1732

[22] Shi H, Andrik J. Aschoff et al. Multislice CT imaging of anomalous arteries, [internet] Eur Radiol, (2004)14: 2172-2181 doi 10.1007/s 00330-604-24902[published online Oct 2004]

[23] Filippo Cademartiri, Ludovico La Grutta, Roberto Malago, Fillipo Alberghina, Francesca Puglies, Erica Maffei et al. Prevalence of Anatomical Variants and Coronary Anomalies in 543 patients studied with 64 slice CT coronary angiography. [internet] Eur Radiol (2008) 18:781-791[online Feb 2008]

[24] Kirsch J., Araoz P. A, Steignberg F. B, Fletcher J.G, McCollough C.H, Williamson E.E, MD.(abstract) Prevalence and Significance of Incidental Extracardiac Findings at Multidetector Coronary CTA. Journal of Thoracic Imaging, Nov 2007-Vol 22-Issue 4-pp 330-334

[25] Lehman, S. J Abbara, S., Cury, R.C., Nagurney, J.T., Hsu, J., Goela, A and Hoffman, U (2009). [Internet] Significance of cardiac Computed Tomography Incidental findings in acute chest pain. (Abstract). The American Journal of Medicine. 122(60), 543-549.

[26] Kim S. Y., Seo J. B. et al. Coronary Artery Anomalies: Classification and ECG-gated Multi-Detector Row CT Findings with Angiographic Correlation. 2006 RadioGraphics, 26, 317-333

[27] Sosnouski, D., Bonsall, R.P, Mayer, F. B and Ravanel, J.G. (2007) Extra cardiac Findings at Cardiac CT: A Practical Approach. Journal of Thoracic Imaging. 22(1), 77-85.

[28] Tamar S, Polonsky, Robyn L et al. Coronary artery calcium score and Risk classification for coronary heart disease prediction, JAMA 2010; 303(16):1610-1616

[29] Budoff M. J, Diamond G A, Raggi P., Arad, Y., Guerci, A.D., Callister, T.Q. and Berman D. (2002) Continuous probabilistic prediction of angiographically significant coronary artery disease using electron beam Tomography, Circulation 105(15), 1791-1796

[30] 30. Jason L. Kelly, David Thickman, Simeon D. Abramson, Pel R. Chen, Stanley F. Smazal et al. Coronary CT angiography findings in patients without coronary calcifications, 2008, vol 191, no 1 JR

[31] Elif Ergun, PinaKosar, Cansu Ozturt, Elif Basbay and Fatma Koc. Prevalence and extent of coronary artery disease determined by 64 slice CTA in patients with zero coronary calcium score, Intl Journal of Cardiovascular imaging, 2011; 27(3) 451-458

[32] Christopher Herzog, Martina Britten, Joern Balzar, Stephan Zangus, Hanns Ackermann et al. European Radiology, 2004;14(2) 169-177

[33] Stampfer M. J, Colditz G.A, Willet W. C, Manson J. E, Rosner $\mathrm{B}$, Speizer $\mathrm{F}$ E et al. Menopause and the risk of coronary heart disease in women. N Engl. J Med 1987; 316(18) 1105-1110

[34] Wilbet S. Aronow, Jerome L. Fleg, Carl J. Pepine, Nancy T. Artinian, George Bakris et al. (2011) ACCF/AHA 2011 Expert Consensus Document on Hypertension in the elderly: A report of the American College of Cardiology Foundation Task Force on clinical Expert Consensus Documents.

[35] Yusuf S. Hawken S. Ounpuu S. Dans T. Anezum A et al. Effect of potentially Modifiable risk factors associated with Myocardial infarction in 52 countries (The INTERHEART Study) Case-control study, The Lancet 2004, 364 (9438); 937-952

[36] Bulugahapitiya U, Siyambalapitiya S, Sithole J and Idris I, Is Diabetes A Coronary Risk Equivalent, A systematic review and meta-analysis, 2009; 26(2);142-148

[37] Haffner S.M, Lehto S., Ronnemaa T. Pyorala K. Laasko M. Mortality from coronary heart disease in subjects with type 2 diabetes and in non-diabetic subjects with and without prior myocardial infarction. N. Engl. J Med 1998, 339; 229-234

[38] Dhamoon M. S, Elkind M.S. Inclusion of stroke as an outcome and risk equivalent in risk scores for primary and secondary prevention of vascular disease. Circulation 2010, 121(18); 2071-2078

[39] Saely CH, Aczel S, Koch L. Schmid F, Marte T, Huber K, Drexel H, DM as Risk Equivalent, (2010) Eur J. Cardiovascular 2010;17(1) 94-99

[40] Shah P.K and R H Helfant, Smoking and coronary artery disease. Chest Journal 1988; 94(3), 449-459

[41] Wang J. C, Normand S. L. T, Mauri L. and Kuntz R. E, Coronary artery spatial distribution of acute myocardial infarction occlusions, Circulation 2004; 110(3); 278-284

[42] Asakura T. and Takeshi Karino. Flow Patterns and Spatial distribution of atherosclerotic lesions in human coronary arteries, Circulation, 1990 66(4); 1045-1066

[43] Svindland Aud, The localization of sudanophilic and fibrous plaques in the main left coronary bifurcation. Atherosclerosis, 1983; 48(2); 139-145 